\title{
Solar Rotational Periodicities and the Semiannual Variation in the Solar Wind, Radiation Belt, and Aurora
}

\author{
Barbara A. Emery • Ian G. Richardson • \\ David S. Evans • Frederick J. Rich • Gordon R. Wilson
}

Received: 17 September 2010 / Accepted: 24 March 2011 / Published online: 12 May 2011

(C) Springer Science+Business Media B.V. 2011

\begin{abstract}
The behavior of a number of solar wind, radiation belt, auroral and geomagnetic parameters is examined during the recent extended solar minimum and previous solar cycles, covering the period from January 1972 to July 2010. This period includes most of the solar minimum between Cycles 23 and 24, which was more extended than recent solar minima, with historically low values of most of these parameters in 2009. Solar rotational periodicities from 5 to 27 days were found from daily averages over 81 days for the parameters. There were very strong 9-day periodicities in many variables in 2005-2008, triggered by recurring corotating high-speed streams (HSS). All rotational amplitudes were relatively large in the descending and early minimum phases of the solar cycle, when HSS are the predominant solar wind structures. There were minima in the amplitudes of all solar rotational periodicities near the end of each solar minimum, as well as at the start of the reversal of the solar magnetic field polarity at solar maximum $(\sim 1980, \sim 1990$, and $\sim 2001)$ when the occurrence frequency of HSS is relatively low. Semiannual equinoctial periodicities, which were relatively strong in the 1995-1997 solar minimum, were found to be primarily the result of the changing amplitudes of the 13.5- and 27-day periodicities, where 13.5-day amplitudes were better correlated with heliospheric daily observations and 27-day
\end{abstract}

The Sun-Earth Connection near Solar Minimum

Guest Editors: M.M. Bisi, B. Emery, and B.J. Thompson

B.A. Emery $(\bowtie)$

HAO/NCAR, 3080 Center Green, Boulder, CO 80301, USA

e-mail: emery@ucar.edu

I.G. Richardson

GSFC and CRESST/Department of Astronomy, University of Maryland, College Park, MD 20742, USA

D.S. Evans

SWPC/NOAA, 325 Broadway, Boulder, CO 80305, USA

F.J. Rich

LL/MIT, 244 Wood St, Lexington, MA 02420-9185, USA

G.R. Wilson

AFRL/RVBXP, 3550 Aberdeen Ave. SE, Kirtland AFB, NM 87117, USA 
amplitudes correlated better with Earth-based daily observations. The equinoctial rotational amplitudes of the Earth-based parameters were probably enhanced by a combination of the Russell-McPherron effect and a reduction in the solar wind-magnetosphere coupling efficiency during solstices. The rotational amplitudes were cross-correlated with each other, where the 27-day amplitudes showed some of the weakest cross-correlations. The rotational amplitudes of the $>2 \mathrm{MeV}$ radiation belt electron number fluxes were progressively weaker from 27- to 5-day periods, showing that processes in the magnetosphere act as a low-pass filter between the solar wind and the radiation belt. The $A_{\mathrm{p}} / K_{\mathrm{p}}$ magnetic currents observed at subauroral latitudes are sensitive to proton auroral precipitation, especially for 9-day and shorter periods, while the $A_{\mathrm{p}} / K_{\mathrm{p}}$ currents are governed by electron auroral precipitation for 13.5- and 27-day periodicities.

Keywords Solar cycle $\cdot$ Solar rotation periodicities $\cdot$ Geospace $\cdot$ Aurora $\cdot$ Radiation belt electron flux $\cdot$ Solar wind $\cdot$ Semiannual periodicity

\section{Introduction}

The solar wind interacts with the Earth's magnetosphere and affects the high-latitude currents and aurora showing that the geospace components are tightly coupled together. In this paper, we demonstrate the influence of the solar wind on geospace by investigating the occurrence of similar periodicities in the solar wind and parameters characterizing the state of the magnetosphere and auroral latitudes on Earth. These periodicities are determined by structures in the solar wind, which vary in character through the solar activity period, and the path of the Earth relative to these structures. Important influences include:

- Solar rotation: The dominant periodicity in the solar wind and many other parameters is the rotation period of the Sun, where the apparent (synodic) rotation as seen at the Earth is $\sim 27$ days at $\sim 25^{\circ}$ heliolatitude near the region of solar active regions. (The true (sidereal) rotation rate of the Sun is $\sim 24$ days at the solar equator increasing to $\sim 30$ days at the poles [see e.g. Howard, 1996; Chandra, Vats, and Iyer, 2009].) Studies of the periodicities in the near-Earth solar wind and geomagnetic indices have found 27-day periods with 'wings' of 25- and 29-day periods (see e.g. Svalgaard and Wilcox, 1975; Gosling et al., 1976, 1977).

- Large-scale structure of the solar wind speed: Around solar minimum, the solar wind has a speed ( $V_{\mathrm{sw}}$ ) of about $400 \mathrm{~km} \mathrm{~s}^{-1}$ at the equator and about $700 \mathrm{~km} \mathrm{~s}^{-1}$ above $30^{\circ}$ of heliomagnetic latitude (see e.g. Mursula and Zieger, 1996; McComas et al., 2003). Around solar maximum, intervals of slow or fast solar wind occur at all latitudes (McComas et al., 2003).

- The configuration of the heliospheric current sheet (HCS) separating magnetic fields from northern and southern heliolatitudes depends on the tilt angle of the solar dipole field with respect to the rotation axis. The dipole field changes sign (polarity) around solar maximum. The tilt varies from $\sim 15^{\circ}$ around solar minimum, resulting in a nearly flat current sheet, to $\sim 75^{\circ}$ around solar maximum, when the current sheet is strongly warped.

- The orbit path of the Earth, which reaches the highest northern heliographic latitude of $+7.2^{\circ}$ on 6 September and the lowest southern heliographic latitude of $-7.2^{\circ}$ on 6 March (see e.g. Cliver, Kamide, and Ling, 2000).

- The Interplanetary Magnetic Field (IMF), where southward IMF (negative IMF, $B_{z}$, in GSM coordinates) results in increased aurora, larger polar-cap potential drops, and stronger currents in the ionosphere (see e.g. Reiff and Luhmann, 1986). The scalar IMF 
magnitude, $B_{\mathrm{t}}$, maximizes in solar maximum, but also shows a temporary decrease related to the reduction in the open magnetic flux associated with the polarity reversal (see e.g. Richardson, Cliver, and Cane, 2000; Richardson, Cane, and Cliver, 2002). The solar wind electric field, $V_{\mathrm{sw}} B_{\mathrm{t}}$, was shown by Emery et al. (2009) to be strongly correlated to the electron auroral global power, $P_{\mathrm{e}}$, for both negative and positive $B_{z}$, where the cross-correlations were strongest for slow-speed wind and high-speed wind streams (HSS) compared to transient solar wind structures.

- Transient solar wind structures associated with Coronal Mass Ejections (CMEs) and their accompanying shocks and compressed sheath regions, most prevalent during solar maximum (Richardson, Cliver, and Cane, 2000; Richardson, Cane, and Cliver, 2002). Such structures are responsible for most of the intense geomagnetic storms (Dst $\leq-100 \mathrm{nT}$ ) seen at Earth (Zhang et al., 2007).

- Polar coronal holes and their accompanying HSS, which expand toward the equator in the declining phase of the cycle, increasing the occurrence frequency of HSS at the Earth, and contract in the ascending phase (see e.g. Emery et al., 2009; Richardson, Cliver, and Cane, 2000; Richardson, Cane, and Cliver, 2002).

Our study is related to that of Mursula and Zieger (1996) who examined the 13.5-day periodicity in solar, heliospheric, and Earth-based parameters. They found that the power spectra of the sunspot number, the Ca K-line plage index, the Geostationary Operational Environmental Satellites (GOES) background X-ray intensity, and the $\mathrm{Mg}$ II core to wing (c/w) ratio showed peaks at the fundamental 27-day periodicity and smaller peaks at the 13.5-day second harmonic. The 13.5-day amplitudes in these solar variables peaked around solar maximum and indicated the presence of two active solar longitudes approximately $180^{\circ}$ apart, as shown in Carrington rotation (27.2753 days) plots. The position of the active solar longitudes could abruptly change by even $90^{\circ}$ between two successive 13.5 -day activations. Only the $\mathrm{Mg}$ II c/w ratio showed a sizable power in the higher harmonics for the solar variables.

Fenimore et al. (1978) found that the 13.5-day periodicity in the solar wind speed could be more dominant than the 27-day periodicity. Mursula and Zieger (1996) showed that the 13.5-day periodicities for $V_{\mathrm{sw}}$ and other heliospheric variables, as well as for the geomagnetic $K_{\mathrm{p}}$ index contained more power than the 27-day periodicities. Higher harmonics (9-, 6.8-, 5.4-, and 4.5-day) were also identified. The 13.5-day power maximized in the late declining phase of the solar cycle, showing that the source of the 13.5-day amplitudes in heliospheric and Earth-based variables was different from the 13.5-day amplitudes in solar variables, which occurred mostly in solar maximum. They attributed the 13.5-day periodicity in the heliospheric variables to a two-stream structure, originating in northern and southern solar latitudes, that the Earth encounters during one solar rotation. Mursula and Zieger (1996) studied the IMF using a coordinate scheme rotated $44^{\circ}$ from the $x$-axis to lie approximately in the direction of the Parker spiral. They called the component aligned with the Parker spiral the IMF "sector" component, or $B_{\mathrm{sr}}$. Unlike the heliospheric plasma parameters, the 27-day power was largest in $B_{\mathrm{sr}}$ and in $B_{z}$ (GSM), and there were clear higher harmonics extending to 4.5-day in $B_{\text {sr }}$, but not in $B_{z}$ (GSM).

The total solar wind input to geospace during storms was estimated by Turner et al. (2009) to be split into the ring current of the magnetosphere $(\sim 10 \%)$, precipitated into the aurora $(\sim 20 \%)$, and dissipated by Joule heating of ions colliding with the neutral atmosphere $(\sim 70 \%)$. The ring current is mostly carried by lower energy ions, while the co-located radiation belt represents mostly higher energy trapped electrons (see e.g. Wolf, 1995). Geostationary satellites orbit near 6.6 Earth radii $\left(R_{\mathrm{E}}\right)$. The GOES satellites measure the number flux of high energy electrons $>2 \mathrm{MeV}$ in the outer radiation belt. Radiation belt electron fluxes decrease during the main phase of magnetic storms and then recover, 
and often increase, during the recovery phase, especially during periods of HSS (Miyoshi and Kataoka, 2005). The increase in electron flux in the recovery phase is particularly effective in the outer radiation belt. The electron fluxes at geosynchronous orbits are wellknown to correlate with the solar wind speed with a lag of 2 or 3 days (Baker et al., 1990; Vassiliadis et al., 2002). We will study the GOES electron number fluxes with energies $>2 \mathrm{MeV}$.

The ionosphere and thermosphere in the upper atmosphere are strongly affected by the solar wind and near-Earth magnetospheric activity, especially under IMF $B_{z}$ (GSM) southward conditions when the Earth's northward magnetic dipole field is easily connected to the IMF southward field. Electric fields in the tail of the magnetosphere are imposed from dawn to dusk in the polar cap of the Earth. The resulting anti-sunward ion convection is closed with sunward return flow in the auroral regions. The aurora ionizes the neutral atmosphere and raises the electrical conductance, thus increasing the Joule heat caused by the difference between the neutral wind patterns and the imposed ion convection.

Knipp, Tobiska, and Emery (2004) showed the high-latitude geomagnetic auroral and Joule power inputs to the Earth to be $\sim 6 \%$ and $\sim 16 \%$ on average, while the solar radiation provides $\sim 78 \%$ of the energy. However, in the top 15 strongest magnetic storms, Joule power provided $\sim 59 \%$ of the total power to Earth, while the auroral power was $\sim 8 \%$. Thus the ratio of auroral power to Joule power is about 1 to 3 on average, but during magnetic storms it decreases to 1 to 4 (see e.g. Turner et al., 2009) and occasionally to 1 to 7. However, these estimates do not include the higher energy ( $>20 \mathrm{keV})$ auroral particles, which for protons can bring in as much energy flux during storms as the lower energies (Fang et al., 2007a), but affect the atmosphere at lower altitudes, since higher energy particles penetrate deeper. We will study the electron and ion auroral hemispheric power inputs with energies $<20 \mathrm{keV}$ estimated from single-pass satellite orbits of the National Oceanic and Atmospheric Administration (NOAA) and Defense Meteorological Satellite Program (DMSP) satellites.

The aurora is composed of precipitating electrons and protons, where protons with energy $<20 \mathrm{keV}$ contribute $\sim 15 \%$ of the total energy flux at $K_{\mathrm{p}}=0$, and $\sim 7 \%$ for $K_{\mathrm{p}}$ values from 4 to 6 (Emery et al., 2008). A small number flux of electrons and protons reaches the dayside polar cap in open field lines from the solar wind, but the majority of the auroral energy flux is deposited in the nightside aurora from the tail of the Earth's magnetosphere. The proton aurora is shifted duskwards from the electron aurora, so that the major impact of proton precipitation in the ionosphere and thermosphere is at the equatorwards edge of the electron aurora in the pre-midnight sector (Galand, Fuller-Rowell, and Codrescu, 2001; Fang et al., 2007b). Newell, Sotirelis, and Wing (2009) estimated the energy flux of the electron aurora to be mostly composed of diffuse precipitation ( $\sim 85 \%$ in quiet times and $\sim 71 \%$ in active times), which enters the Earth's atmosphere from the magnetosphere through pitchangle scattering (see e.g. Wolf, 1995). The balance of the electron precipitation is in broadband electrons from dispersive Alfvén waves and monoenergetic electrons that are accelerated and enter the atmosphere to produce arcs and other auroral forms with a great deal of structure. The structured ("discrete") electron auroral forms create strong gradients in the resulting ionization and conductance, which strongly affect currents. Newell, Sotirelis, and Wing (2009) estimated the proton aurora to be diffuse, precipitating from the magnetosphere through pitch-angle scattering and charge exchange with neutrals (see e.g. Wolf, 1995), where Mende et al. (2002) reviewed four processes in the inner magnetosphere that can disturb the proton pitch-angle distribution and lead to enhanced proton precipitation. Estimated loss times for electrons from the inner plasma sheet are typically a few hours, while the loss time is much longer for ions because they are heavier in mass and take much longer 
to complete a bounce between hemispheres along a magnetic field line, where each mirroring point is an opportunity to precipitate into the atmosphere through pitch-angle diffusion (see e.g. Wolf, 1995). The different time constants and precipitation forms for electron and proton aurora indicate that their periodic characteristics may be different.

Emery et al. (2009) examined the solar wind structure sources and periodicities of auroral electron power $\left(P_{\mathrm{e}}\right), V_{\mathrm{sw}}$, and IMF $B_{\mathrm{t}}$ from 1978 to 2008 . This work used an updated version of the list of solar wind structures created by Richardson, Cliver, and Cane (2000) and Richardson, Cane, and Cliver (2002) based on in-situ solar wind observations, as well as related effects such as geomagnetic storm sudden commencements and cosmic ray depressions. The solar wind structures are separated into HSS, slow-speed wind, transients associated with interplanetary coronal mass ejections (ICMEs), and unknown structures when observations were lacking. As previously noted by Richardson, Cliver, and Cane (2000) and Richardson, Cane, and Cliver (2002), the fraction of the solar wind at 1 AU occupied by transients peaks during solar maximum and minimizes during solar minimum. HSS from coronal holes maximize in the descending phases of the solar cycle and minimize at the end of solar minima and around solar maxima, while slow-speed winds fill out the rest of the solar wind structure. Emery et al. (2009) found that the time variation of the solar wind velocity $V_{\mathrm{sw}}$ and the auroral electron power $P_{\mathrm{e}}$ are determined by the HSS, which contribute $\sim 47 \%$ to the total $V_{\mathrm{sw}}$ and $P_{\mathrm{e}}$. Transients contribute $\sim 42 \%$ to $P_{\mathrm{e}}$ in solar maxima and $\sim 6 \%$ in solar minimum. The solar rotational periodicities in $P_{\mathrm{e}}$ were similar to those of $V_{\mathrm{sw}}$ with strong 9-day amplitudes in 2005 and 2008, strong 7-day amplitudes in 2006, and large equinox semiannual amplitudes in $1995-1999$.

Gibson et al. (2009) confirmed the 9-day periodicity of the Whole Heliospheric Interval (WHI $N^{\circ}$ ) Carrington rotation in 2008 in $V_{\mathrm{sw}}$ and $P_{\mathrm{e}}$, and showed it also existed in the $>2 \mathrm{MeV}$ electron number flux from the geostationary satellite GOES-12 in the radiation belt near 6.6 Earth radii $\left(R_{\mathrm{E}}\right)$. They contrasted the coronal hole and HSS variations in WHI with the Whole Sun Month (WSM) solar minimum Carrington rotation in 1996 where the 9-day periodicity was absent in these heliospheric, magnetospheric, and Earth-based parameters.

The 9-day periodicity in 2005 and 2008 seen in the solar wind, the electron auroral power, and the radiation belt electron flux at $6.6 R_{\mathrm{E}}$ (for 2008), was also seen in the neutral density at $400 \mathrm{~km}$ (Lei et al., 2008a, 2008b, 2011), in the total electron content (TEC) (Lei et al., 2008c), in the polar thermospheric neutral winds (Lei et al., 2008c), and in the power radiated through infrared cooling by $\mathrm{CO}_{2}$ and $\mathrm{NO}$ between 100 and $200 \mathrm{~km}$ (Mlynczak et al., 2008, 2010). The 20067 -d peak was observed again in these same thermospheric quantities by Lei et al. (2008b, 2008c), Thayer et al. (2008), and Mlynczak et al. (2010). The 9-day variations were not seen in the $10.7 \mathrm{~cm}$ solar flux or in the EUV flux from the Solar EUV Experiment (SEE) onboard the NASA TIMED (Thermosphere-Ionosphere-Mesosphere Energetics and Dynamics) satellite (Lei et al., 2008b). The lack of 9-day variations in the EUV or $10.7 \mathrm{~cm}$ solar flux is not surprising because the 9-day variations are a function of the solar magnetic field geometry, and not a photon effect.

The aim of this study is to examine the behavior of a number of solar wind (heliospheric), radiation belt (magnetospheric), auroral and geomagnetic (Earth-based) parameters during the recent extended solar minimum and previous solar cycles, covering the period from January 1972 to July 2010. We will examine the minima in these parameters and compare them over four solar cycles. In particular, we will focus on solar rotational periodicities in these parameters, where 'rotational' in this study refers not only to the fundamental 27-day period, but also the subharmonics with shorter periods. We will also study the semiannual periodicities, and cross-correlations between and among the parameters, and their periodic amplitudes. The auroral indices and electron number flux $>2 \mathrm{MeV}$ observed in the radiation 
belt are only available in the last three solar minima, but we extend our study to Solar Cycle (SC) 20 to gain insight into the differences in these four solar cycles. Cross-correlations between the amplitudes of different periodicities can help determine if the source of the periodicities in the heliospheric parameters is the same or not compared to the magnetospheric and Earth-based parameters. Time variations and other changes in the amplitudes are also of interest in determining how the solar wind is filtered through the magnetosphere to the high-latitude regions.

\section{Data}

We use hourly values of the solar wind velocity and IMF from the OMNI2 data set, available from the Space Physics Data Facility at http://omniweb.gsfc.nasa.gov/ow.html, which also supplies values of the 3-hour $a_{\mathrm{p}}$ and $K_{\mathrm{p}}$ geomagnetic indices from the National Geophysical Data Center (NGDC) in Boulder, Colorado. The $K_{\mathrm{p}}$ index is found from 13 selected subauroral stations (originally 11 ) from $-43^{\circ}$ to $-47^{\circ}$ magnetic latitude (mlat) in the southern hemisphere and from 49 to 62 mlat in the northern hemisphere. The 3-hour $a_{\mathrm{p}}$ index is a linearized form of the logarithmic 3-hour $K_{\mathrm{p}}$ index. The daily average of eight 3-hour $a_{\mathrm{p}}$ indices is referred to as $A_{\mathrm{p}}$.

Daily medians and averages were computed from the hourly averages. The magnitudes of periodicities of 5 days and longer in these data (as discussed below) were found to be approximately the same using daily or hourly averaged data, so the present study used daily averages of all quantities.

The hourly list of solar wind structures compiled by Richardson, Cliver, and Cane (2000) and Richardson, Cane, and Cliver (2002) was updated for this study. Daily 'averages' were used where a day was called 'transient' if any hour contained a transient. If a day had HSS and slow-speed wind, it was called 'HSS'.

Carrington rotation averages of the tilt angle of the heliospheric current sheet (HCS) from May 1976 to July 2010 were taken from the Wilcox Solar Observatory website at http://wso. stanford.edu/Tilts.html ("line of sight" values are used). Earlier values were inferred from the inclination of the solar dipole field to the ecliptic (Wang, Sheeley, and Rich, 2000; Wibberenz, Richardson, and Cane, 2002). The HCS tilt angle is usually found from a solar magnetic field map over a single rotation period. Thus, we were not able to find rotational periodicities in this data set because tilt observations were only available every Carrington rotation.

Radiation belt electron number fluxes from the GOES satellites come from NGDC via the Space Physics Interactive Data Resource (SPIDR) at http://spidr.ngdc.noaa.gov starting with GOES-05 in January 1986 and extending through GOES-12. The GOES satellites orbit around the equator at geostationary heights of $\sim 6.6 R_{\mathrm{E}}$ (Earth radii, or L-shell 6.6). Daily averages were used to eliminate significant diurnal variations resulting from the satellite locations. The GOES-06 number fluxes were considerably lower than observations from the other GOES satellites and affected by sensor degradations at the end of its lifetime in $1992-1994$ from comparisons of its periodic amplitudes. GOES-06 data were only used from 86001 to 87084 over the same time period as all the GOES-05 data, which appeared to be high. The GOES-07 data, which started in 87065, were in line with later GOES-08 data, and were used when the GOES-06 data were deleted after 87084. The resulting daily average number fluxes from one to three GOES satellites were used in this study.

The equatorward boundary of precipitating auroral electrons at midnight or the Auroral Boundary Index (ABI) (Gussenhoven, Hardy, and Burke, 1981; Gussenhoven, Hardy, and 
Heinemann, 1983) is estimated from DMSP satellites and is available starting with DMSPF06 from December 1982 from the Coupling, Energetics and Dynamics of Atmospheric Regions (CEDAR) Database (DB) at http://cedarweb.hao.ucar.edu/wiki/index.php/DMSP:Main. In quiet periods, the midnight equatorward boundary is closer to the pole, while during active periods, it moves equatorwards. Fang et al. (2007a) compared the ABI electron boundary to the equivalent proton equatorward boundary at midnight from NOAA satellite $30-240 \mathrm{keV}$ protons $\left(\mathrm{ABI}_{\mathrm{H}+}\right)$ and Imager for Magnetopause-to-Aurora Global Exploration (IMAGE) satellite FUV-SI12 protons for a sheath-driven and saw-tooth magnetic cloud storm (both CME transients) in April 2002 in their Figure 12. ABI $\mathrm{H}_{+}$was usually equatorwards of ABI as expected. Most of the time it was within $1 \mathrm{deg}$, but during the recovery of the sheathdriven storm, the proton boundary was $4^{\circ}$ equatorwards of the electron boundary because electrons have a much faster decay time than ions. The proton equatorward boundary on the dusk side at 18 Magnetic Local Time (MLT) is $\sim 5^{\circ}$ equatorwards of the equivalent electron boundary (see e.g. Galand, Fuller-Rowell, and Codrescu, 2001). This is because electrons precipitate into the atmosphere in the poleward Region I upward Field-Aligned Currents (FACs), while protons precipitate into the equatorward Region II downward FACs on the duskside (see e.g. Wolf, 1995).

The total hemispheric power $\left(\mathrm{HP}_{\mathrm{t}}\right)$ of precipitating auroral particles was estimated by Fuller-Rowell and Evans (1987) using in-situ ion and electron observations from NOAA Television and Infrared Observation Satellites (TIROS) on a background of statistical patterns of the electron and ion precipitation based on 10 levels of the estimated $\mathrm{HP}_{\mathrm{t}}$. These estimates are available from http://www.swpc.noaa.gov/ftpmenu/lists/hpi.html. This method was adapted for use with the electron detectors on the DMSP satellites to produce estimates of electron hemispheric power $\left(\mathrm{HP}_{\mathrm{e}}\right)$ available from the CEDAR DB. Emery et al. (2005, 2006, 2008) estimated the NOAA HP $\mathrm{e}_{\mathrm{e}}$ using only the in-situ electron particle detectors and intercalibrated the NOAA $\mathrm{HP}_{\mathrm{e}}$ and $\mathrm{HP}_{\mathrm{t}}$ estimates with the DMSP $\mathrm{HP}_{\mathrm{e}}$ estimates to produce a single set of hourly and daily $\mathrm{HP}_{\mathrm{e}}$ estimates available at the CEDAR DB at http://cedarweb. hao.ucar.edu/wiki/index.php/Instruments:ehp. The intersatellite calibration was revised after DMSP estimates of $\mathrm{HP}_{\mathrm{e}}$ were recalibrated to adjust for instrument degradation on counts through the South Atlantic Anomaly region as used in the study of Hardy et al. (2008) which removed sunlight contamination and other problems.

The corresponding NOAA ion hemispheric power $\left(\mathrm{HP}_{\mathrm{i}}\right)$ was found as the difference between the total and electron hemispheric powers $\left(\mathrm{HP}_{\mathrm{i}}=\mathrm{HP}_{\mathrm{t}}-\mathrm{HP}_{\mathrm{e}}\right)$ and was also intercalibrated among the available NOAA Space Environment Monitor 2 (SEM-2) satellites (Emery et al., 2006, 2008) where the baseline was set by estimates of HPi from DMSP satellites by Hardy, Gussenhoven, and Brautigam (1989). A complete re-calibration was done for this study after $\mathrm{HP}_{\mathrm{e}}$ (and $\mathrm{HP}_{\mathrm{i}}$ ) estimates became available from SEM-1 NOAA satellites starting in 1982, where the SEM-1 HP $\mathrm{H}_{\mathrm{i}}$ magnitudes between 1982 and 1988 were adjusted using ratios of $\mathrm{HP}_{\mathrm{i}}$ with $\mathrm{HP}_{\mathrm{e}}$ and $A_{\mathrm{p}}$. Emery et al. $(2006,2008)$ estimated that most of $\mathrm{HP}_{\mathrm{e}}$ was carried by electrons with energies $<20 \mathrm{keV}$ because estimates from the Medium Energy Proton and Electron Detector (MEPED) NOAA instruments at energies between $30-240 \mathrm{keV}$ account for $<10 \%$ of the electron energy flux. However, approximately half the ion energy flux is from ions with energy $>20 \mathrm{keV}$ for $K_{\mathrm{p}}$ levels of 4 or more from a study by Fang et al. (2007a) as interpreted by Emery et al. (2008).

For this study, we will use the total global power estimated from the sum of HP from each hemisphere for electrons $\left(P_{\mathrm{e}}=\mathrm{HP}_{\mathrm{e}}(\mathrm{SH})+\mathrm{HP}_{\mathrm{e}}(\mathrm{NH})\right)$ and ions $\left(P_{\mathrm{i}}=\mathrm{HP}_{\mathrm{i}}(\mathrm{SH})+\mathrm{HP}_{\mathrm{i}}(\mathrm{NH})\right)$. Where only one satellite gives a measurement in a single UT hour, the global power is estimated at twice the single hemisphere available. Daily average $P_{\mathrm{e}}$ and $P_{\mathrm{i}}$ calculated from hourly average $P_{\mathrm{e}}$ and $P_{\mathrm{i}}$ are used in this study. Emery et al. (2008) showed that $\mathrm{HP}_{\mathrm{e}}$ and $\mathrm{HP}_{\mathrm{i}}$ increase with $K_{\mathrm{p}}, A_{\mathrm{p}}$, and $V_{\mathrm{sw}}$, and both increase strongly with increasing $B_{z}$ (GSM) 
negative. $\mathrm{HP}_{\mathrm{i}}$ also increases strongly with $B_{z}$ increasing positive, while the increase in $\mathrm{HP}_{\mathrm{e}}$ is weak with $B_{z}$ positive. Emery et al. (2009) found significant cross-correlations of $P_{\mathrm{e}}$ with various combinations of $V_{\mathrm{sw}}$ and IMF, where a good correlation for both positive and negative $B_{\mathrm{z}}$ conditions was found with the solar wind electric field, or $V_{\mathrm{sw}} B_{\mathrm{t}}$, where $B_{\mathrm{t}}$ is the total magnitude of the IMF.

Figure 1 shows 27-day Bartels rotation averages of various solar, solar wind, radiation belt and auroral parameters from 1972 to 2010 . These include the $10.7 \mathrm{~cm}$ solar flux (a close proxy for the sunspot number), the tilt angle of the HCS, the azimuthal $(\Phi)$ angle of the IMF (GSE coordinates), the percentage of time in different solar wind structures (ICMEs and related structures, HSS, and slow solar wind), solar wind speed, density and dynamic pressure, IMF intensity $\left(B_{\mathrm{t}}\right)$, and the solar wind electric field $\left(\mathrm{VB}_{\mathrm{t}}\right)$ plotted in the same panel as the Kan-Lee merging electric field (Kan and Lee, 1979). The radiation belt $>2 \mathrm{MeV}$ average electron number flux measured by available GOES spacecraft is shown below the solar wind parameters, while the auroral parameters illustrated include the 0 MLT auroral equatorward boundary (ABI) in magnetic latitude, and the global electron and ion auroral power inputs $P_{\mathrm{e}}$ and $P_{\mathrm{i}}$. The bottom panel shows the geomagnetic index $A_{\mathrm{p}}$.

\section{Historic Minimum Parameter Values}

As is evident from Figure 1, many solar wind parameters change over the solar cycle, including the magnitude of the IMF and the tilt angle of the HCS, which tend to follow the level of solar activity. The solar rotation averages of the azimuthal angle $\left(\tan \phi_{\mathrm{B}}=-B_{y} / B_{x}\right)$ of the IMF indicate the dominant field direction in each rotation. Because of the direction of the global solar magnetic field, which reverses near solar maximum, and the Earth's orbital motion, $\phi_{\mathrm{B}}$ is more likely to be inward $\left(\sim 315^{\circ}\right)$ in the early part of the year from 1972 to 1979 , when the Earth is south of the solar equator and more likely to encounter inward fields below the HCS, then switches to outward $\left(\sim 135^{\circ}\right)$ in the early part of the year from 1980 to 1989, when fields below the HCS are outward after the solar field reversal at the maximum of SC 21 in $\sim 1980$. The pattern reverses again from 1990 to 1997. The situation is very mixed from 1998 to 2006, partly because of the high tilt angle of the HCS (see the second panel of Figure 1) but from 2007 to 2009, the pattern has again reversed, as expected following the solar field reversal at the maximum of SC 23. The pattern in $\phi_{\mathrm{B}}$ is quite clean at the end of each solar minimum period because the tilt angle is low, and the HCS is relatively flat such that the heliolatitude of the Earth is the main factor determining the predominant direction of the IMF at the Earth.

In Figure 1, the solar minima periods (shaded) are defined by a smoothed monthly sunspot number less than 20, similar to Maris and Maris (2005). The last solar minimum, between SC 23 and 24, was longer and deeper than the previous minima shown in the figure. This figure only shows data through July 2010, but the solar minimum period extended at least through September 2010. We have calculated sliding 27-day averages of each parameter to find the lowest 27-day minima for each SC, and have plotted these minima as red circles. We define the start of each solar cycle as starting 150 days into the ascending phase of each separate cycle because the minima in the parameters are often found in or just after the shaded official solar minima period. Additional minima from sliding 81-day averages (red squares) or sliding 27-day medians of daily medians (red triangles) are plotted if they occur more than 81 days from the red circles. All variables show some kind of minima in 2008 or 2009. The dashed horizontal lines go through approximate minima for the three previous solar cycles. Note that most quantities drop well below these dashed lines starting around 2006 (see e.g. McComas et al., 2008, for discussion of the solar wind parameters), 


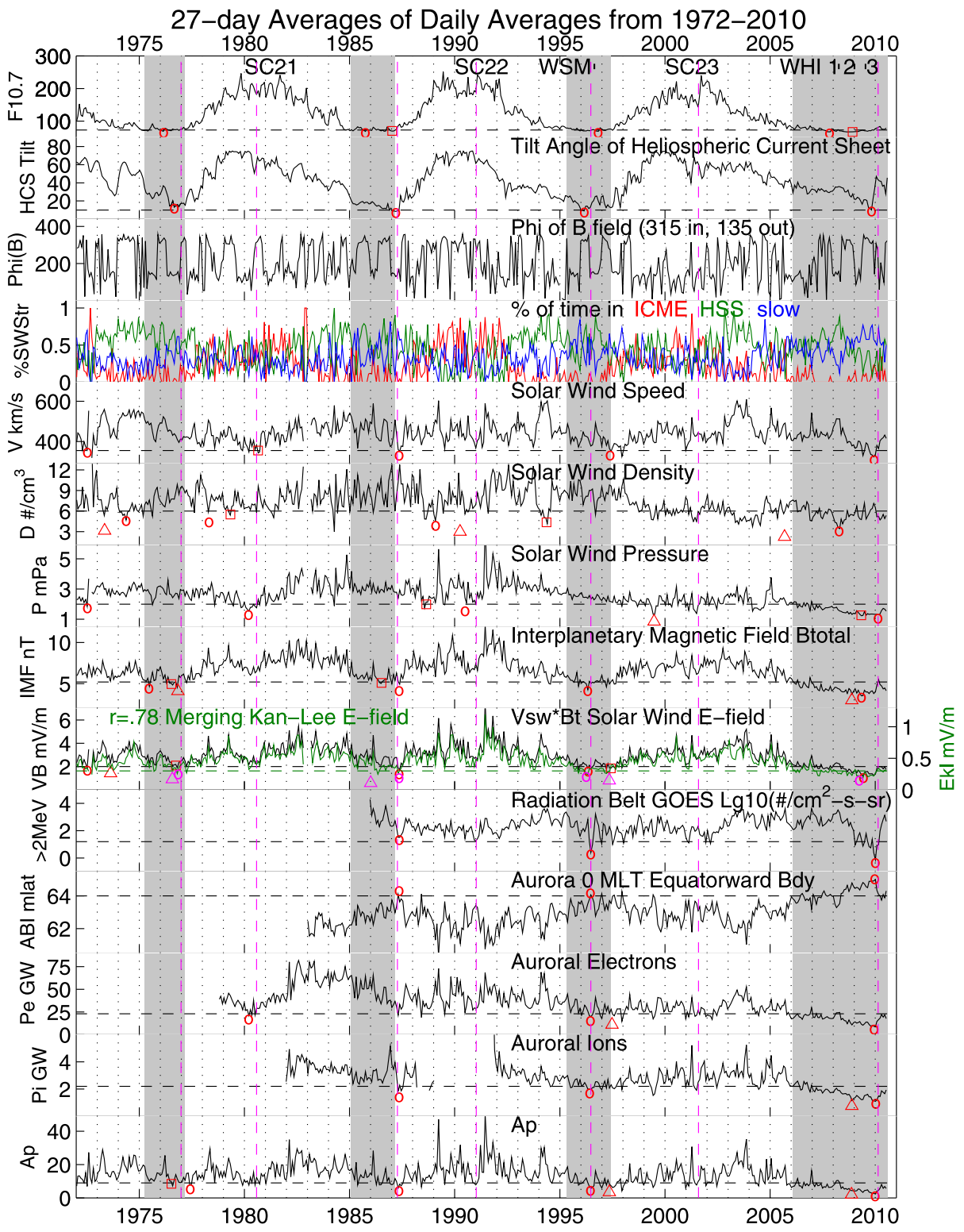

Figure 1 27-day (Bartels rotation) averages from 1972 to 2010 for various solar wind and geophysical parameters. Sliding 27-day average minima are plotted as red circles for each solar cycle (SC) where the start and end of each SC is defined to be 150 days into the ascending phase. When the sliding 81-day average minima (red squares) or the sliding 27-day median minima from daily medians (red triangles) occur more than 81 days from the red circles, they are also plotted. The horizontal dashed lines are the approximate minimum values before 2005 to illustrate the depth of the present solar minimum. The vertical magenta dashed lines mark approximate locations where the solar rotation amplitudes are at a minimum at the end of each solar minimum and at the time of the polarity reversal in the solar magnetic field. 
demonstrating that the minima in 2009 are historic for this period of study. Most quantities minimize at the end of each solar minimum period, (or maximize in the case of the equatorward boundary of the midnight auroral oval, $\mathrm{ABI})$, except for the solar wind density $\left(D_{\mathrm{sw}}\right)$ and pressure $\left(P_{\mathrm{sw}}\right)$. The density is anti-correlated with the solar wind speed especially in solar minimum as seen in Figure 1, when the solar wind alternates between slow dense flow from the HCS and tenuous HSS. The solar wind density often minimizes in the ascending or descending phases of the solar cycle, while the pressure usually minimizes during solar cycle maximum (see e.g. Richardson and Kasper, 2008), as shown in Figure 1 for 1980 and 1990 before the onset of HSS increases for the solar wind pressure. Even the 27-day average solar wind density and pressure minimize in solar minimum during SC 23, and these are the deepest minima in the period 1972-2010. However, the minima in the 27-day medians (red triangles) occurred in 2005 and 1999 for $D_{\mathrm{sw}}$ and $P_{\mathrm{sw}}$, outside the solar minimum period and more in line with the minima during the other solar cycles. The HCS tilt angle, however, was lower in 1987 and 1996 than in 2009 and persisted at higher values for much of the recent solar minimum, rather than falling to low values as in previous minima.

Transients (in the fourth panel in red), which include ICMEs and the associated shocks and post-shock flows, maximize in solar maximum when they are present for $\sim 35 \%$ of the time (Richardson, Cane, and Cliver, 2002; Emery et al., 2009). The ICME contributions shown in Figure 1 are somewhat larger because any day that includes a transient is flagged as a transient day in the daily structure index. The contribution of HSS (in green) maximizes in the descending and initial solar minimum phases, and is smallest at the end of solar minimum and during solar maximum. The remaining slow-speed solar wind (in blue) fills in and is more prevalent in late solar minimum periods. The slow-speed solar wind always has some component that originates from the streamer belt near the $\mathrm{HCS}$ with low $\mathrm{He} / \mathrm{H}$ abundances of $\sim 1 \%$, while other slow-speed wind sources come from higher heliolatitudes where the $\mathrm{He} / \mathrm{H}$ abundance is $\sim 4 \%$ (Richardson and Kasper, 2008; Aellig, Lazarus, and Steinberg, 2001).

The solar wind electric field, $\mathrm{VB}_{\mathrm{t}}$, in Figure 1 has a similar time dependence to the merging Kan-Lee electric field $\left(E_{\mathrm{kl}}=V_{\mathrm{sw}} B_{\mathrm{tan}} \sin ^{2}(\theta c / 2)\right.$, where $\theta c=\arctan \left(B_{y} / B_{z}\right)$ and $B_{\tan }=\sqrt{\left(B_{y}^{2}+B_{z}^{2}\right)}$ using GSM $B_{y}$ and $B_{z}$ coordinates; Kan and Lee, 1979), although the Kan-Lee field is about five times smaller in the 27-day averages made from daily averages. Daily and hourly auroral power inputs for electrons $\left(P_{\mathrm{e}}\right)$ and ions $\left(P_{\mathrm{i}}\right)$ are well correlated with the solar wind electric field, as shown by Emery et al. (2009), as are the $K_{\mathrm{p}}$ and $a_{\mathrm{p}}$ geomagnetic indices (Emery et al., 2008). Emery et al. (2009) showed that the cross-correlations of $\mathrm{VB}_{\mathrm{t}}$ with $P_{\mathrm{e}}$ were particularly strong for periods when HSS were present. Gibson et al. (2009) also showed that $P_{\mathrm{e}}$ was well correlated with $V_{\mathrm{sw}}$ in magnitude, until $P_{\mathrm{e}}$ dropped lower than expected based on $V_{\mathrm{sw}}$ because of the decrease in the IMF magnitude $B_{\mathrm{t}}$ starting in 2007. $P_{\mathrm{e}}$ is also highly anti-correlated with the related index of the equatorward boundary of the auroral oval at midnight or the Auroral Boundary Index (ABI), where $r=-0.89$ for the daily values $\left(P_{\mathrm{e}} \sim \max [0.2,976.9-14.962 * \mathrm{ABI}]\right)$. The ABI reached a historic poleward position of $65.12^{\circ}$ in magnetic latitude in 27-day averages on day $354 \pm 13$ days of 2009. Most parameters in Figure 1 increased sharply starting in January 2010, signaling the end of this historic, long solar minimum period.

\section{Solar Rotational Periodicities}

\subsection{Amplitudes}

Previously, Emery et al. (2009) showed that the 27-day period and subharmonic 7-, 9-, and 13.5-day amplitudes were largest for $P_{\mathrm{e}}$ and $V_{\mathrm{sw}}$ in the descending and minimum phases of 
the solar cycle based on yearly amplitudes. Gibson et al. (2009) showed for the first Whole Heliospheric Interval (WHI $\mathrm{N}^{\circ} 1$ ) in 2008 that the radiation belt electron fluxes for $>2 \mathrm{MeV}$ fluxes had the same periodicities as the solar wind speed and the electron auroral power, with strong 9-day amplitudes. The 9-day periodicity in 2008 was also seen in the neutral density at $400 \mathrm{~km}$ (Lei et al., 2011), in infrared cooling between 100 and $250 \mathrm{~km}$, and in the $K_{\mathrm{p}}$ index, but it was not present in the $10.7 \mathrm{~cm}$ solar flux (Mlynczak et al., 2010). The 9-day periodicity of HSS and other geospace parameters during WHI $\mathrm{N}^{\circ} 1$ in 2008 was based on two low-latitude coronal holes in the north and south hemispheres of the Sun, followed by winds from the polar coronal hole in the southern hemisphere (see e.g. Gibson et al., 2009).

We calculate the periodic properties of the parameters plotted in Figure 1 using the Lomb-Scargle (Lomb, 1976; Scargle, 1982) periodogram technique for unequally spaced data. The Lomb-Scargle periodogram is a form of least-squares spectral analysis (LSSA) [http://en.wikipedia.org/wiki/Least-squares_spectral_analysis] that estimates a frequency spectrum, similar to Fourier analysis, using least-squares fits of sinusoids to unequally spaced data. The square root of the power level in a Lomb-Scargle periodogram, or its amplitude, is similar to the Fourier amplitude [http://sigspec.org/manual/node49.html]. The Lomb-Scargle method mitigates the problems of Fourier analysis, and has other desirable properties such as better definition of peak amplitudes in time or frequency domain.

Figures $2 b-c$ plot the daily peak Lomb-Scargle amplitudes near the 27- and 13.5-day periods from 1972 to 2010 over sliding 81 day analysis periods, while Figure 2a plots the amplitudes of all the rotational periods with different colors. Because of the close correlation of the equatorward electron auroral boundary ABI and $P_{\mathrm{e}}$, they are plotted on the same panel in Figures $2 b-c$, while ABI is eliminated in Figure 2a. Similarly, the amplitudes of the absolute values of $B_{y}$ and $B_{z}$ (components of the IMF) are plotted with $B_{\mathrm{t}}$ (the total IMF magnitude) in Figures $2 \mathrm{~b}-\mathrm{c}$, but are eliminated in Figure 2a. The Kan-Lee merging electric field amplitudes are plotted separately from the solar wind electric field $\left(\mathrm{VB}_{\mathrm{t}}\right)$ amplitudes. For the radiation belt electron number flux, the amplitudes of $\log 10$ of the number flux are plotted. All the other amplitudes plotted in Figures $2 \mathrm{a}-\mathrm{c}$ are absolute amplitudes, not relative amplitudes, and thus reflect the magnitudes of the original data. Therefore, the relatively large amplitudes in 1991 reflect in part the large magnitudes of these quantities shown in Figure 1 for this solar maximum period.

In general, the largest amplitudes in Figure 2a occur in the descending and minimum phases, with the notable exception of large amplitudes in all periods during the maximum of SC 22 in 1989 and 1991, similar to the large amplitudes found by Emery et al. (2009) in 1991 in their Figure 6. From Figure 1, the tilt angle of the HCS was large at this time, which is normal for solar maximum. The $\phi_{\mathrm{B}}$ angle, especially for 1991, shows large variations typical of two-stream flow with high HCS tilt angles. In addition, the active regions for CMEs were confined to a single solar longitude (Richardson, Mazur, and Mason, 1998), which imposed an additional 27-day periodicity to the transient activity during this SC 22 solar maximum.

In Figure 2a, the amplitudes in $A_{\mathrm{p}}, V_{\mathrm{sw}}, P_{\mathrm{sw}}$, and other parameters are weakest at the end of each solar minimum period, where $B_{\mathrm{t}}$ is also minimum (see Figure 1), and at the temporary depressions in $B_{\mathrm{t}}$ during each solar maximum $(\sim 1979-1980, \sim 1990$, and $\sim 2001$ in Figure 1) near the beginning of the reversal of the magnetic field polarity (see e.g. Richardson, Cliver, and Cane, 2000; Richardson, Cane, and Cliver, 2002). These intervals, when the periodicities are weakest, are indicated by dashed magenta lines in Figures 1 and 2 and were chosen somewhat arbitrarily on the basis of low amplitudes in $A_{\mathrm{p}}$ and $V_{\mathrm{sw}}$. Often, the amplitudes of periodicities in $B_{\mathrm{t}}$ and related quantities are relatively significant as seen in Figure 2a during these intervals when the amplitudes of other parameters are quite low. This 


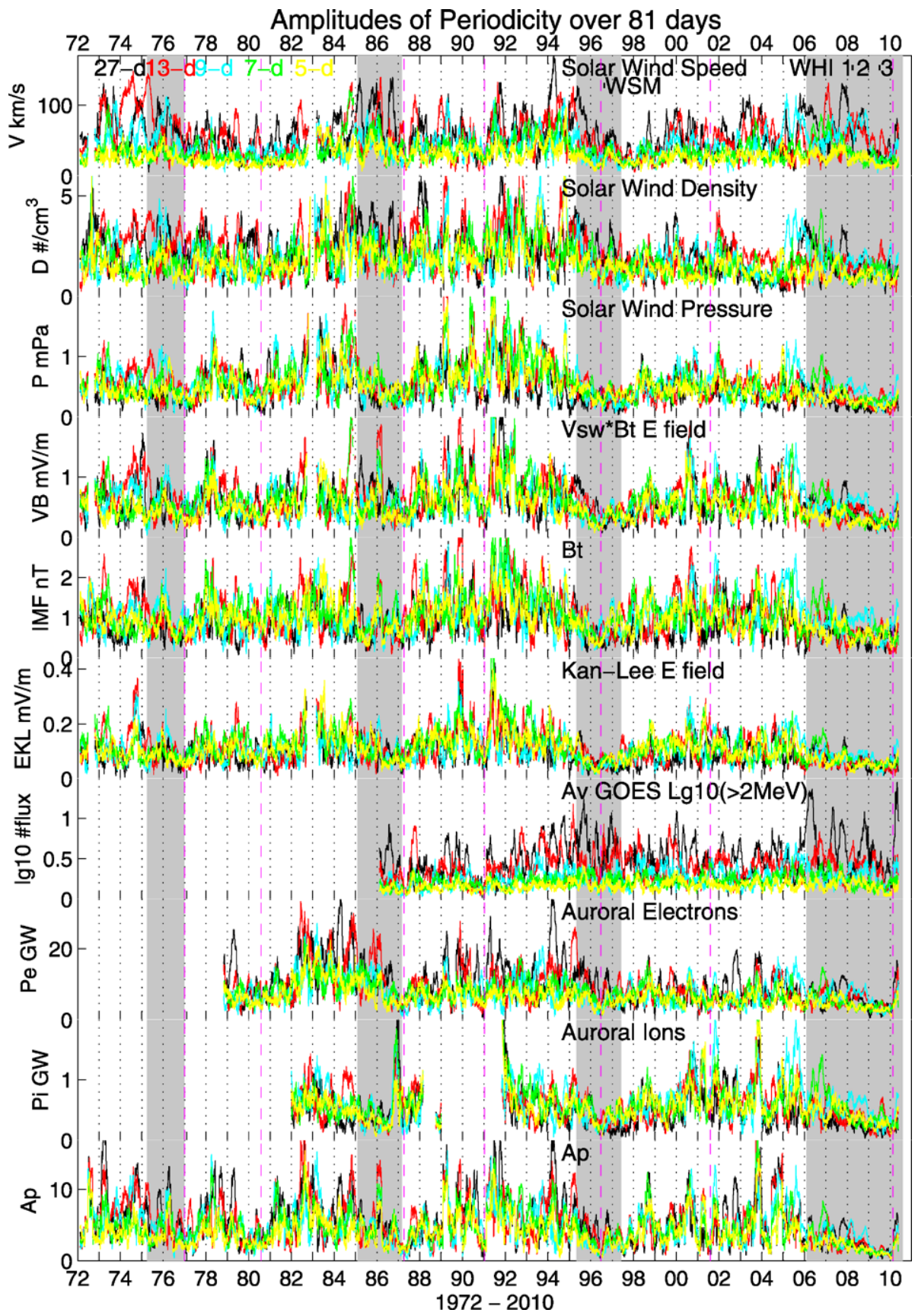

(a)

Figure 2 27-day averages of the daily sliding 81-day amplitudes in the solar wind parameters, radiation belt $>2 \mathrm{MeV}$ electron number flux, auroral indices, and $A_{\mathrm{p}}$ for periods of (a) $27.0 \pm 3.0$ days (black), $13.5 \pm 1.0$ or 1.5 days (magenta), $9.0 \pm 0.5$ days (cyan), $6.85 \pm 0.75$ days (green), and $5.4 \pm 0.4$ days (yellow). (b) $27.0 \pm 3.0$ days and (c) $13.5 \pm 1.0$ or 1.5 days. Magenta dashed lines show the approximate times during solar minimum and maximum where the amplitudes are relatively low. The correlation coefficients are between the sliding 81-day daily, (b) 27-day, or (c) 13.5-day amplitudes of ABI and $P_{\mathrm{e}}$. 


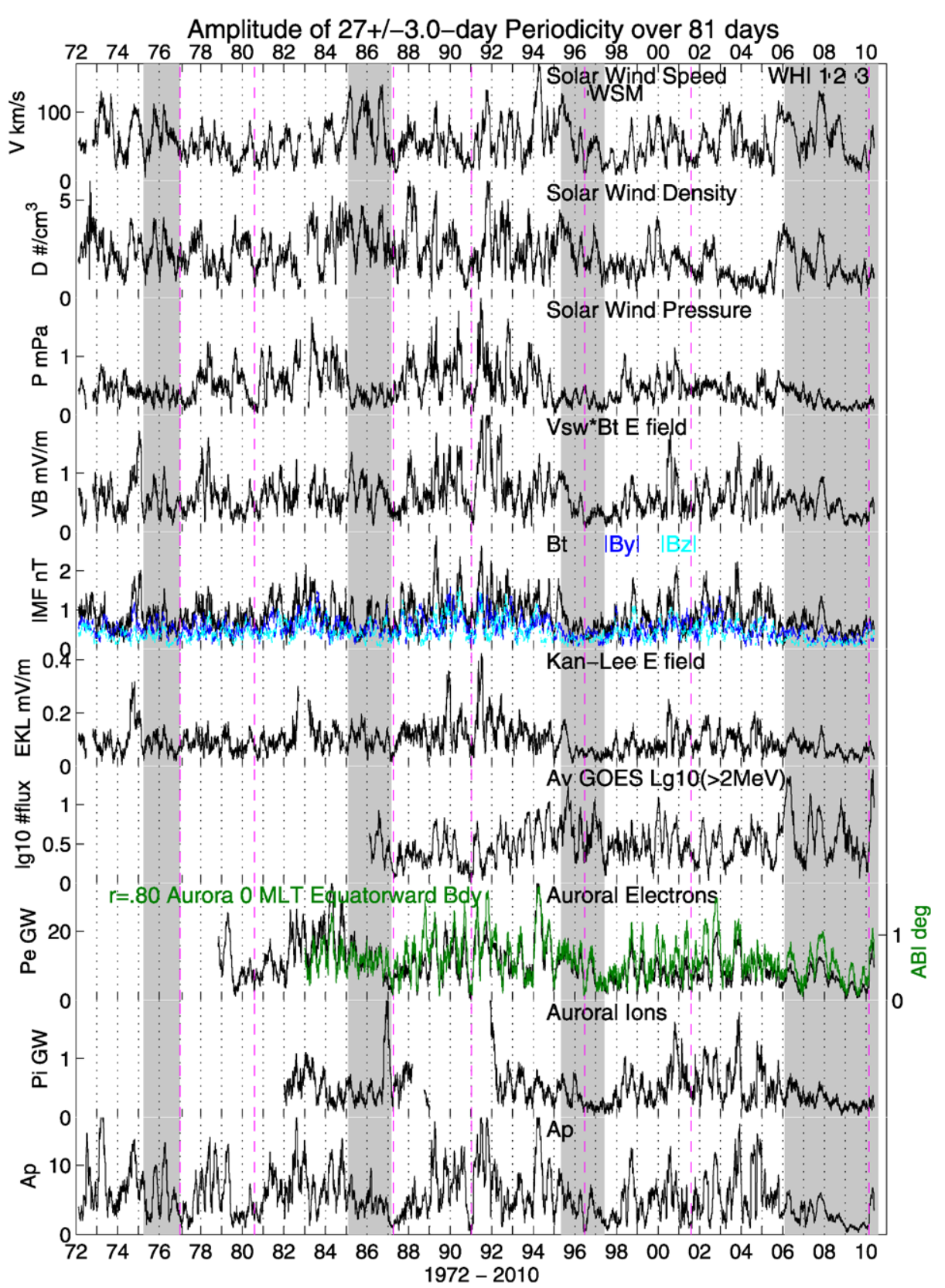

(b)

Figure 2 (Continued)

could indicate that the total solar field is more variable when it is reorganizing. The dipole axis of the solar magnetic field is approximately aligned with the solar rotation axis during solar minimum, but then rotates to the other pole each successive maximum, crossing the 


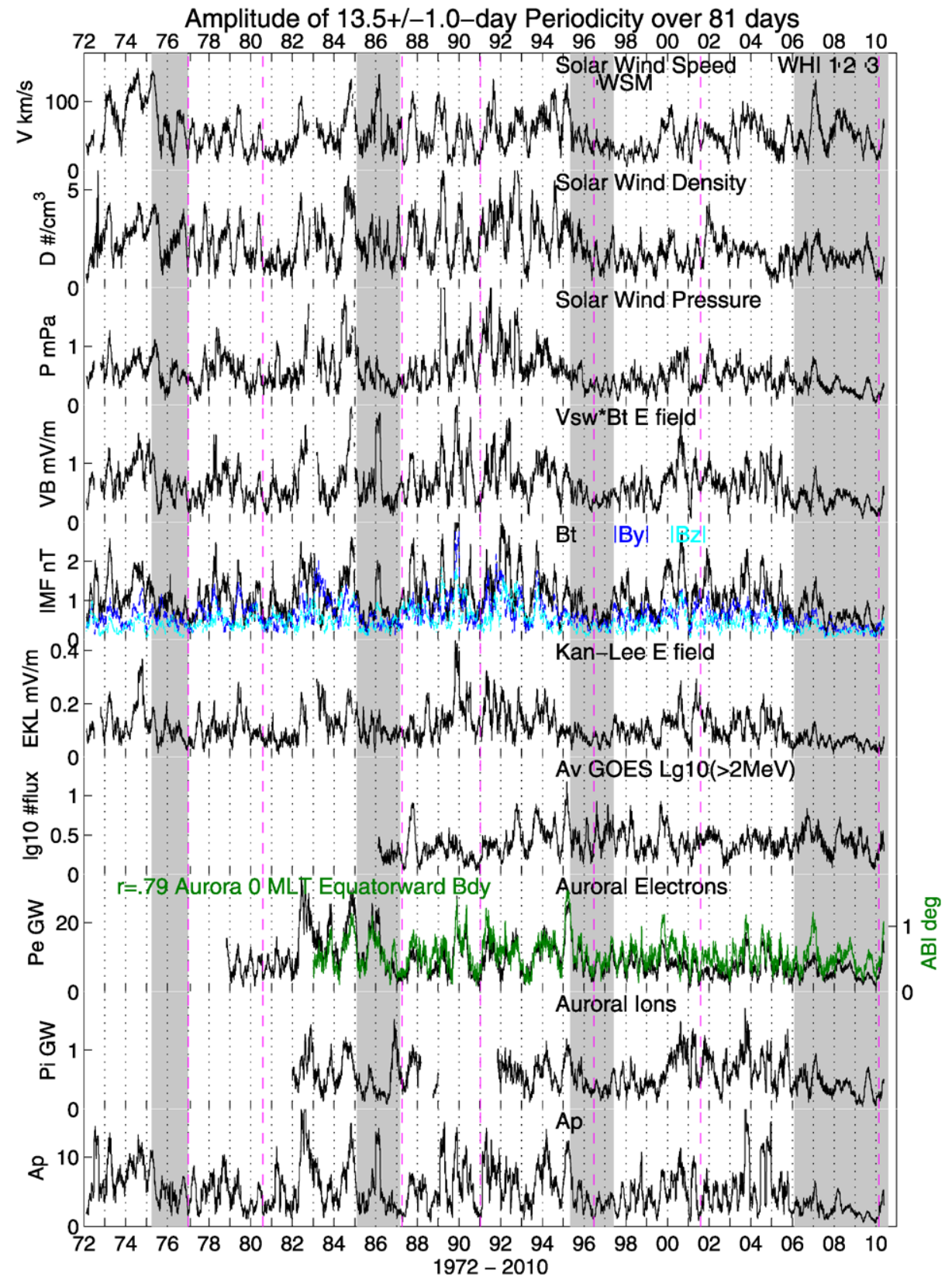

(c)

Figure 2 (Continued)

equator and changing polarity. Many of these rotational periodicities are associated with the solar magnetic field and with the way the Earth intersects this field above and below the HCS. Therefore, when the solar magnetic field is weak or re-organizing as seen at $1 \mathrm{AU}$, 
that could be why the rotational amplitudes in the solar wind and associated parameters at the Earth are also weak. However, the 13.5-day amplitude is mostly the result of two-stream flow, which is particularly effective when the HCS tilt is low at the end of each solar minimum period. The 13.5-day amplitude peaks once or twice per year, and it is not clear how these peaks are associated with the HCS tilt. It is unfortunate that there are no daily HCS tilt angle values to be able to calculate the rotational period amplitudes to see how they compare in particular with the 13.5-day amplitudes of other heliospheric parameters at $1 \mathrm{AU}$.

Figure 2a shows that the amplitudes of these rotational periodicities are approximately of the same magnitude for the heliospheric quantities related to the IMF $B_{\mathrm{t}}$, although the magenta color shows above the top of the $B_{\mathrm{t}}$ amplitudes, indicating that the 13.5-day amplitudes might be larger than the others. Mursula and Zieger (1996) studied the IMF sector component $B_{\text {sr }}$ in the $x-y$ plane and found the 27-day power spectrum magnitude (amplitude) was largest for $B_{\mathrm{sr}}$ and for $B_{z}(\mathrm{GSM})$, while the 13.5-day amplitude was largest for the IMF radial magnitude $B_{\mathrm{r}}$. $B_{\mathrm{sr}}$ had clear spectral peaks from 27- to 4.5-day, while the peaks in $B_{z}$ and $B_{\mathrm{r}}$ were weak. Larger peaks in the black 27-day amplitudes and the magenta 13.5-day amplitudes are apparent in $P_{\mathrm{e}}$ and $A_{\mathrm{p}}$, while the auroral ion input power, $P_{\mathrm{i}}$, shows magnitudes of all the periods to be about the same, indicating that the characteristics of the pitch-angle diffusion of low energy $(<20 \mathrm{keV})$ protons into the atmosphere is different from that for electrons, which have faster time constants and additional acceleration mechanisms for discrete electrons. Both $V_{\mathrm{sw}}$ and $D_{\mathrm{sw}}$ show a preference for larger magnitudes in the 27- and 13.5-day periods, but $P_{\mathrm{sw}}\left(P_{\mathrm{sw}}=D_{\mathrm{sw}} V_{\mathrm{sw}}^{2} / N_{\mathrm{o}}\right)$ shows magnitudes that are about the same for all periods because $D_{\mathrm{sw}}$ and $V_{\mathrm{sw}}$ are often anti-correlated (Richardson and Kasper, 2008).

The amplitudes of the $>2 \mathrm{MeV}$ radiation belt electron number flux show a clear decrease in magnitude from the fundamental 27-day period to each subsequent subharmonic in Figure $2 \mathrm{a}$. This clearly demonstrates that the radiation belt is the result of processes that filter the solar wind through a low-pass filter, where higher frequencies (shorter time periods) are attenuated. Baker et al. (1990) showed that during a period in $1982-1985$ with significant HSS, the geostationary energetic electron fluxes with energies $>0.5 \mathrm{keV}$ at $6.6 R_{\mathrm{E}}$ had strong auto-correlation peaks at 27 days with secondary peaks at 13 days from HSS, as well as at 54 and 81 days. The $\log$ of the electron fluxes were best correlated with the solar wind speed with a 2 day lag, and with $K_{\mathrm{p}}$ and AE with a 3 day lag. Vassiliadis et al. (2002) showed that the cross-correlation of the $\log$ of the electron flux measured at $\sim 7.25 R_{\mathrm{E}}$ and $V_{\mathrm{sw}}$ maximized at $\sim 50 \%$ for a lag $\sim 2.5$ days from their Figure 1. Lag times increased closer to the Earth, so at $6.6 R_{\mathrm{E}}$, the lag was nearly 3 days with cross-correlations slightly less than $50 \%$. This delay time includes the growth time of ULF waves as well as the time delay of 2 days between the electron flux increases and ULF waves found by Rostoker, Skone, and Baker (1998) in their Figure 1. Vassiliadis et al. (2002) also estimated the effective magnetospheric "memory time" of the processes determining the electron flux to previous solar wind inputs. This time is somewhere between 11 days and 21 days, with 21 days giving a better fit to the observed fluxes. The duration of the response function for the electron number flux is $\sim 6$ days at $6.6 R_{\mathrm{E}}$ at solar maximum and $\sim 9$ days at solar minimum ( $\sim 15-18$ days at $\left.4 R_{\mathrm{E}}\right)$, which could explain why the 9-day and shorter amplitudes in the energetic electron fluxes at $6.6 R_{\mathrm{E}}$ are small as they are less than the "memory time".

The variations in the amplitudes of these periodicities found in the solar wind parameters often carry through to the Earth-based parameters, such as: the large 27-day black amplitudes in 1973, 1985, 1994, 2007, and 2010; the large 13.5-day magenta amplitudes in 1974, 1982, 1984, 1986, 1987, and 1995; the large 9-day cyan amplitudes in 1976, 1989, 1993, and 2005; the large 6.85-day green amplitudes in 1973, 1981, 1984, and 2006; and the large 
5.4-day yellow amplitudes in 1983 and 1994. The 27-day amplitudes in the heliospheric solar wind quantities in Figures $2 \mathrm{a}$ and $2 \mathrm{~b}$ do not carry through as well to the Earth-based parameters, partly because of geometrical effects that enhance equinoctial periods that will be discussed later in the section on semiannual periodicities. The large 13.5-day amplitudes in Figure 2c are stronger in the Earth-based parameters in late 1983 compared to the solar wind parameters, while in early 2007, the reverse is true (except for a large peak amplitude in $\mathrm{ABI})$.

Considering previous solar cycles, the longest interval with particularly enhanced 13.5day amplitudes in $V_{\text {sw }}$ in Figure 2c occurred in 1974-early 1975. This is also when the tilt angle of the HCS rose unexpectedly to $\sim 60^{\circ}$ (second panel of Figure 1) after first descending towards low values more typical of solar minimum. At this time, the Earth experienced exceptionally strong two-stream solar wind flows from extensions of the northern and southern polar coronal holes (see e.g. Sheeley, Harvey, and Feldman, 1976), leading to the strong 13.5-day amplitudes in the solar wind. This is consistent with the conclusion of Mursula and Zieger (1996) that the 13.5-day amplitudes in the heliospheric and $K_{\mathrm{p}}$ variables result from the two-stream structures that the Earth intersects when the HCS is sufficiently flat and tilted.

\subsection{Cross-Correlations}

Figures $3 \mathrm{a}-\mathrm{h}$ plot the cross-correlations of the daily Lomb-Scargle amplitudes between all the parameters for the 'independent' solar wind parameters $V_{\mathrm{sw}}, D_{\mathrm{sw}}$, and $B_{\mathrm{t}}(3 \mathrm{a}-\mathrm{c})$, radiation belt $>2 \mathrm{MeV}$ electron fluxes (3d), and the Earth-based parameters of ABI, $P_{\mathrm{e}}, P_{\mathrm{i}}$, and $A_{\mathrm{p}}(3 \mathrm{e}-\mathrm{h})$. The cross-correlations between the daily observations plotted in Figure 1 are also plotted in Figures $3 \mathrm{a}-\mathrm{h}$ on the right-hand side of the plots. These are "normalized" cross-correlations so that the auto-correlation at zero-lag is 1.0. The formula for the "normalized" cross-correlations is the same as the cross-correlations used by Vassiliadis et al. (2002) in their Equation (1). Normally, a cross-correlation is considered significant if it is larger than 0.50 , so this level is marked with a dotted black line in Figures $3 a-h$. Crosscorrelations with the solar wind quantities are in purple and red, while cross-correlations with Earth-based parameters are in blue for the radiation belt, dark green for the aurora, and black for $A_{\mathrm{p}}$. Because of the inherent lag time of 2-3 days in the electron fluxes in the outer radiation belt at $6.6 R_{\mathrm{E}}$, the cross-correlations in Figure $3 \mathrm{~d}$ are all made with a 2 day lag of the $>2 \mathrm{MeV}$ electron fluxes, with the other plots showing cross-correlations with 0 and 3 day lags as well.

Figures $3 \mathrm{a}-\mathrm{c}$ concentrate on the solar wind parameters $V_{\mathrm{sw}}, D_{\mathrm{sw}}$ and $B_{\mathrm{t}}$. The 27-day amplitudes do not correlate as well as the 5-day through 13.5-day amplitudes. The best cross-correlations are with $B_{\mathrm{t}}$ in Figure $3 \mathrm{c}$, where cross-correlations with $\mathrm{VB}_{\mathrm{t}}, P_{\mathrm{i}}$, KanLee E (Ekl), $A_{\mathrm{p}}$, and $P_{\mathrm{sw}}$ are all above $50 \%$ for all amplitudes and totals. The best total cross-correlation of the daily $V_{\mathrm{sw}}$ values in Figure $3 \mathrm{a}$ is with the $\log$ of the electron number flux at geosynchronous altitudes with a 2 day lag followed by the anticorrelation of the equatorward boundary at 0 MLT (ABI).

It is apparent in Figure $3 \mathrm{~d}$ that only the solar wind speed correlates well with the radiation belt $>2 \mathrm{MeV}$ electron number flux with a 2 day lag having a slightly better cross-correlation than a 3 day lag as in Baker et al. (1990). The next best total cross-correlations with the $>2 \mathrm{MeV}$ fluxes are with $P_{\mathrm{e}}$, the auroral equatorward boundary $\mathrm{ABI}$, and $A_{\mathrm{p}}$ with 3 day lags having slightly better cross-correlations than 2 day lags in Figures $3 \mathrm{e}-\mathrm{g}$, consistent with the 3 day lag found by Baker et al. (1990) for $K_{\mathrm{p}}$. The total cross-correlations are better with a 2 or 3 day lag for $V_{\text {sw }}$ in Figure 3a, and for all the auroral parameters in Figures $3 \mathrm{e}-\mathrm{h}$. 

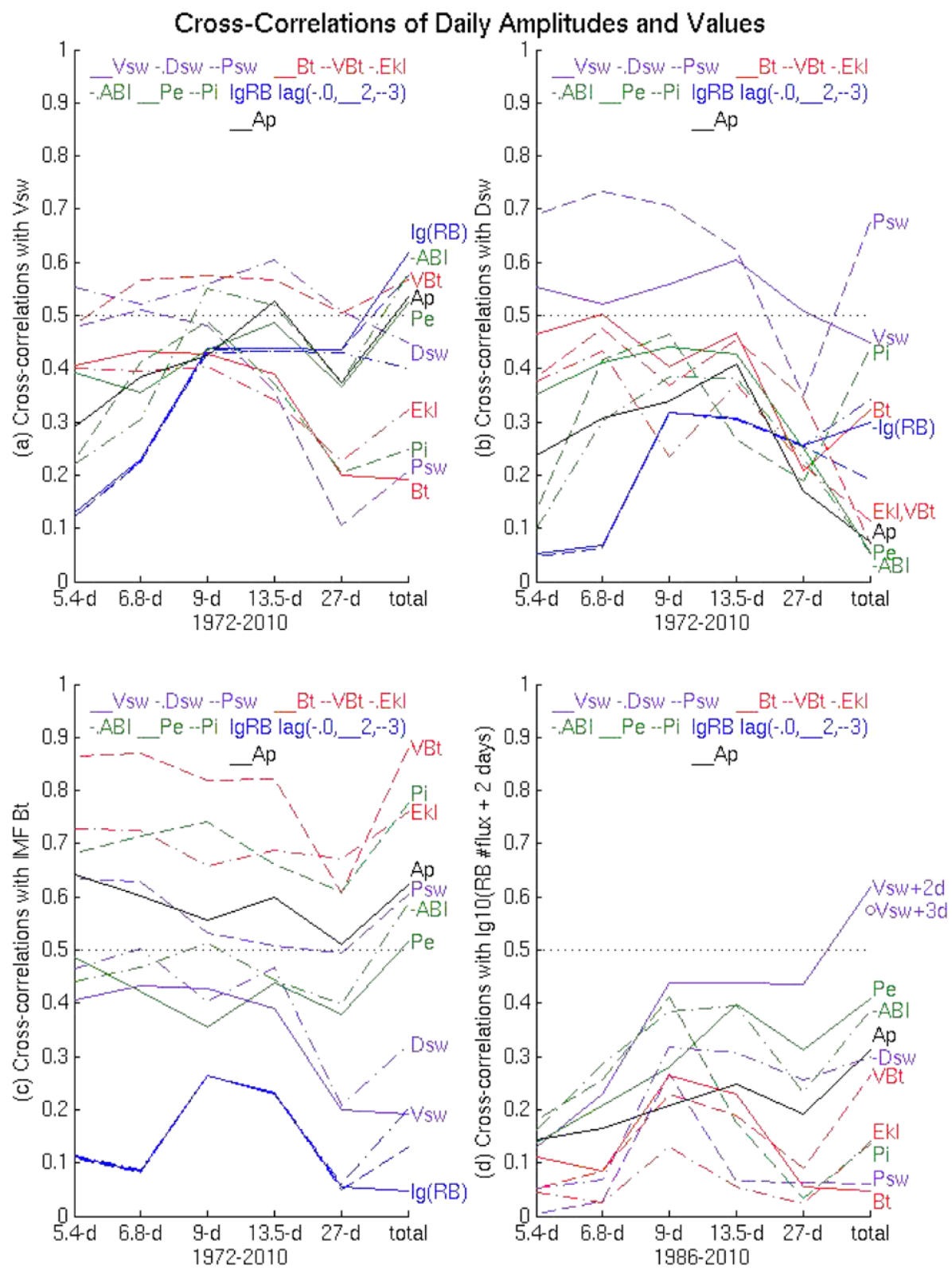

$(a-d)$

Figure 3 Total daily cross-correlations and cross-correlations of the daily sliding 81-day amplitudes from Figure 2a between solar wind parameters (purple and red), radiation belt $>2 \mathrm{MeV}$ electron number fluxes (blue), auroral indices (dark green), and $A_{\mathrm{p}}$ (black) for (a) $V_{\mathrm{sw}}$, (b) $D_{\mathrm{sw}}$, (c) $B_{\mathrm{t}}$, (d) radiation belt $>2 \mathrm{MeV}$ electron number fluxes, (e) ABI, (f) $P_{\mathrm{e}}$, (g) $P_{\mathrm{i}}$, and (h) $A_{\mathrm{p}}$. The $>2 \mathrm{MeV}$ fluxes are lagged 2 days from the other parameters with additional lags of 0 days and 3 days shown. 

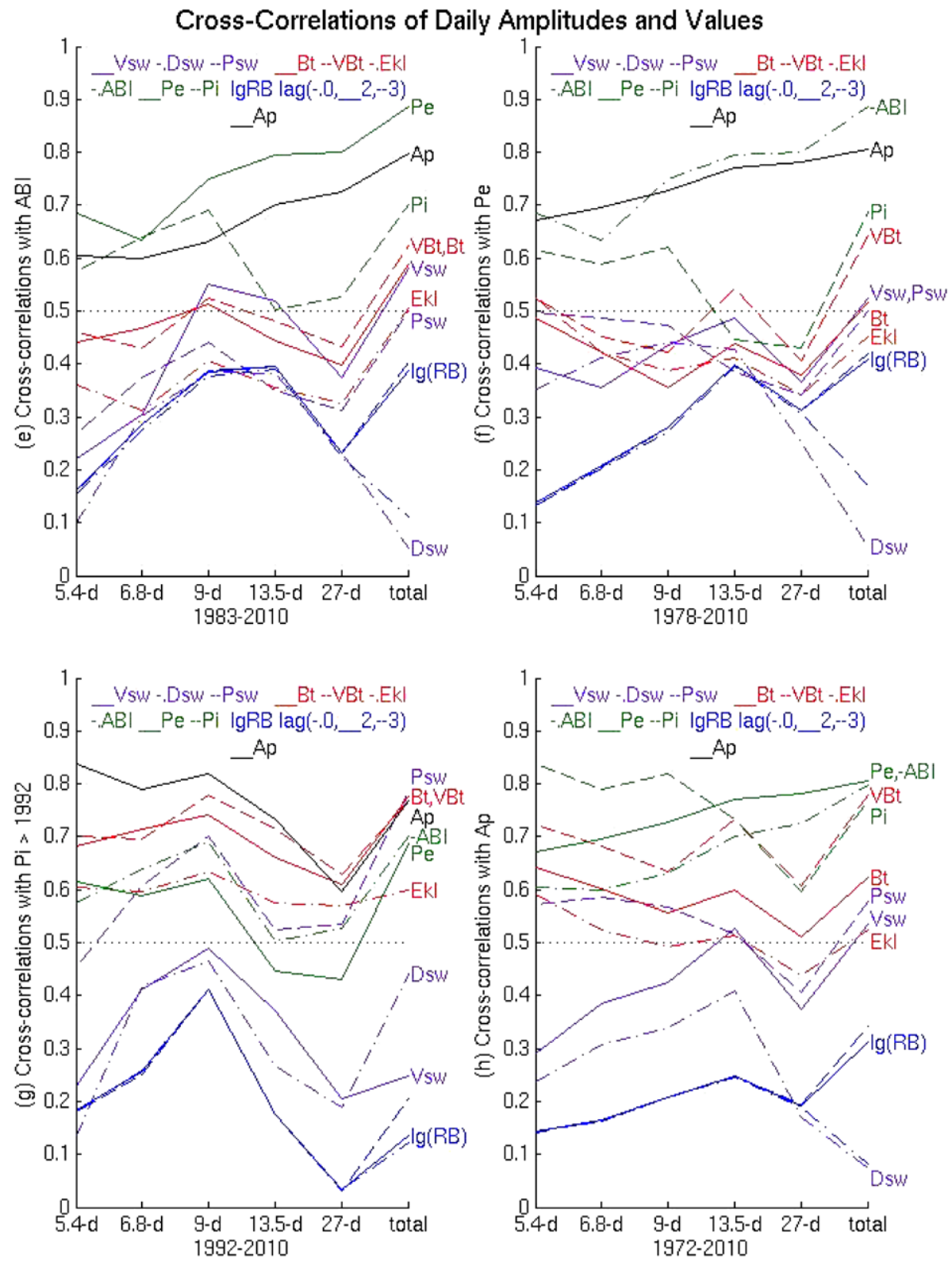

$(\mathrm{e}-\mathrm{h})$

Figure 3 (Continued)

However, a 0 day lag (dot-dashed blue line) is better for $D_{\mathrm{sw}}$ and $B_{\mathrm{t}}$ in Figures $3 \mathrm{~b}-\mathrm{c}$, and a 0 day lag with $P_{\mathrm{i}}$ in Figure $3 \mathrm{~g}$ is not much worse than a 2 day lag. The $P_{\mathrm{i}}$ results could reflect the longer time it takes protons to pitch-angle diffuse into the atmosphere after the solar wind plasma is swept into to magnetosphere. The cross-correlations for the 5- and 7- 
day amplitudes of the $>2 \mathrm{MeV}$ electron flux are particularly low, since they are strongly attenuated in the radiation belt, as explained earlier.

Figures $3 \mathrm{e}-\mathrm{h}$ show that the 27-day amplitudes are less correlated than the subharmonic amplitudes, except that the correlations among $\mathrm{ABI}, P_{\mathrm{e}}$ and $A_{\mathrm{p}}$ increase from 5-day through 27-day amplitudes to maximize in the total cross-correlations. Unlike the other parameters, the ion auroral power $P_{\mathrm{i}}$ in Figure $3 \mathrm{~g}$ shows relatively poor cross-correlations with the 13.5$\mathrm{d}$ amplitudes. Indeed, the highest cross-correlations for $A_{\mathrm{p}}$ in the 5-d through 9-d amplitudes are with $P_{\mathrm{i}}$ in Figure $3 \mathrm{~h}$, switching to $P_{\mathrm{e}}$ for the total and for 13.5-d and 27-d amplitudes. The stronger sensitivity to proton aurora in the subauroral $A_{\mathrm{p}}$ index could be related to the fact that proton precipitation pushes the auroral boundary equatorwards between $15-21$ MLT from $\sim 67$ mlat to $\sim 61$ mlat in the $K_{\mathrm{p}} 3$ simulation by Galand, Fuller-Rowell, and Codrescu (2001). Since the $K_{\mathrm{p}}$ stations are located between 43 and $62^{\circ}$ mlat, the equatorward expansion of the auroral currents should affect the subauroral $K_{\mathrm{p}}$ latitudes proportionately more than the $\sim 15 \%$ global increase in auroral energy flux and $\sim 10 \%$ increase in the Joule heating found for this simulation.

\section{Semiannual Periodicities}

We now consider semiannual periodicities. In Figure 1, the semiannual period appears to be strong in the SC 22/23 minimum from 1995 to 1997 in $V_{\mathrm{sw}}$ (and inversely in $D_{\mathrm{sw}}$ ), in the radiation belt $>2 \mathrm{MeV}$ electron flux, in the auroral parameters, and in $A_{\mathrm{p}}$. A possible contributing factor is illustrated in Figure 2c, where there are peaks in the 13.5-day amplitudes for all these parameters around the time of the equinoxes. The 27-day amplitudes in Figure $2 \mathrm{~b}$ for $V_{\mathrm{sw}}$ were also strong around April in 1995 and 1996, and in January in 1997, while the equinoctial peaks in the Earth-based parameters tended to be equally strong in March and September in 1995-1997.

In Figure 2b, there seem to be strong semiannual variations in the 27-day amplitudes of $P_{\mathrm{e}}$ most years between 1983 and 2002. There are similar equinoctial peaks in the 27-day amplitudes of the $6.6 R_{\mathrm{E}}$ electron number flux, $P_{\mathrm{i}}$, and $A_{\mathrm{p}}$, which are not always present in the solar wind variables. For instance, there is usually one peak per year in $V_{\mathrm{sw}}$ and its 27-day amplitudes from 1977 - 1984 while there are usually two peaks per year in $A_{\mathrm{p}}$ and its 27-day amplitudes in the same time period. Another example is in 1990 where there are deep minima in the 27-day amplitudes of most parameters at the end of the year, but there are strong equinoctial peaks in $A_{\mathrm{p}}$ and $P_{\mathrm{e}}$ while there are peaks in January 1990 in $V_{\mathrm{sw}}$ and in June 1990 in $B_{\mathrm{t}}$. The Kan-Lee merging electric field is the only solar wind parameter with small equinoctial components in addition to the large solsticial 27-day amplitudes in January and June, but $A_{\mathrm{p}}$ and $P_{\mathrm{e}}$ show minima in January, July (in $P_{\mathrm{e}}$ and $\mathrm{ABI}$ ), and in December in their corresponding 27-day amplitudes for 1990.

Cliver, Kamide, and Ling (2000) discussed the origin of the semiannual periodicity in the am geomagnetic index as due to three separate effects. The first is the axial effect due to the variation in the heliolatiude of the Earth along its orbit, contributing $\sim 15-20 \%$ of the semiannual periodicity in $\mathrm{am}$.

The second effect on the semiannual amplitude of am and other related quantities such as $A_{\mathrm{p}}$ and the auroral inputs is the Russell-McPherron effect (Russell and McPherron, 1973) where geometry favors more negative $B_{z}$ in GSM coordinates in the higher heliolatitudes near equinoxes compared to the solstices. This effect should be seen in the Kan-Lee merging electric field results of this study. However, the cross-correlations of the Ekl merging electric field with $P_{\mathrm{e}}$ and $P_{\mathrm{i}}$ are lower than the cross-correlations of the solar electric field $\mathrm{VB}_{\mathrm{t}}$ as seen in Figures $3 \mathrm{e}$ and $3 \mathrm{f}$. 
The third effect on the semiannual amplitude of am was ascribed by Cliver, Kamide, and Ling (2000) to a loss of coupling efficiency that reduces the flow of solar wind into the Earth's system during solstices. This would affect the Earth-based parameters but not the solar wind parameters so the Earth-based would see larger semiannual variations. Emery et al. (2009) found that the semiannual equinoctial amplitude for $P_{\mathrm{e}}$ was of $\sim 15.7 \%$ variation, while the $V_{\text {sw }}$ equinoctial amplitude was of $\sim 3.0 \%$ variation.

We suggest that the strong semiannual periodicity in $V_{\mathrm{sw}}$ and the Earth-based parameters is rooted in the combination of the semiannual variations in the 13.5- and 27-day amplitudes. To demonstrate this, Figure 4 is made similar to Figure 1, but the dark green lines show the result of reconstructing the parameters using just the 13.5- and 27-day amplitudes from Figures $2 b$ and $2 c$, using as a baseline the values minus these amplitudes on day 48 of 2010 , a period of low rotational amplitudes, marked by a dashed magenta line. It is apparent that most of the structure in these parameters can be captured by including just the 13.5- and 27day amplitude variations. Cross-correlations of the daily total values in black with the daily sum of the 13.5- and 27-day amplitude variations in dark green are less than 0.50 and range from 0.24 for $P_{\mathrm{sw}}$ to 0.40 for $P_{\mathrm{e}}$. The cross-correlations were less for the 13.5- and 27-day amplitudes alone, where the solar wind parameter observations corresponded better to their 13.5-day amplitudes and the Earth-based parameter daily observations corresponded better to their 27-day amplitudes. The cross-correlations only increased minimally after adding the 9-day or smaller period amplitudes to the more significant 13.5- and 27-day amplitudes. The correspondences in Figure 4 are suggestive, but the cross-correlations are not significant, so we calculate the semiannual variation directly from the original daily values (black lines) and the sum of the baseline plus 13.5- and 27-day amplitudes (dark green lines) in Figure 4.

Figure 5 shows the Lomb-Scargle amplitudes of the (183 \pm 17 )-day (semiannual) periodicity for the daily parameters plotted in Figure 4 calculated from 567-day sliding blocks advanced every 81 days. The amplitudes are only plotted if there is a peak within 166-200 days $(183 \pm 17)$ days. The phase of the semiannual variation is not given in the LombScargle technique, so some of these peaks could be solsticial instead of equinoctial. The semiannual amplitudes from the sum of the 13.5- and 27-day amplitudes (dark green $x$ 's) show the same general trends as the real amplitudes from the daily averages (black circles), except possibly in the absolute magnitude of the IMF $\left|B_{y}\right|$ in GSM coordinates.

There are significant peaks at all times within the solar cycle, where some of the larger peaks outside the solar minimum periods are in 1982-1983, 1989-1990, 2001-2002, and 2004. Some of these peaks may reflect the magnitude of the values plotted in Figure 4 at these times, such as the peaks in the Kan-Lee electric field, but the sum of the 13.5- and 27day amplitudes shown as dark green lines in Figure 4 do not reflect the solar cycle variations as much as the original data do.

The largest semiannual amplitudes in $A_{\mathrm{p}}$ are outside of solar minimum in 1973, 1989 1990, and 2001, where there are relatively large equinoctial peaks in $A_{\mathrm{p}}$ in Figures 1 and 4. The $A_{\mathrm{p}}$ peaks in 1989-1990 and 2001 are primarily the result of equinoctial peaks in the ICME frequency in red in the fourth panel of Figure 1, which results in especially strong peaks in $V_{\mathrm{sw}}, B_{\mathrm{t}}$ and related parameters around March 1989 and March 2001 even though not all of the ICMEs were geoeffective. Semiannual peaks in $P_{\mathrm{sw}}$ are especially large in $1989-1990$ as are the Earth-based semiannual peaks in $P_{\mathrm{e}}$, the equatorward auroral boundary (ABI), and $A_{\mathrm{p}}$. The $\mathrm{VB}_{\mathrm{t}}$ semiannual peaks are relatively large in 1973 and 2001. The semiannual amplitudes of $A_{\mathrm{p}}$ correlate best $(r=0.78)$ with those of the ion auroral power, $P_{\mathrm{i}}$, as was also the case for most of the rotational and subharmonic amplitudes as shown in Figure $3 \mathrm{~h}$.

The highest semiannual amplitudes in $V_{\mathrm{sw}}$ occur in solar minimum 1986, where there are two large equinoctial peaks in $V_{\mathrm{sw}}$ in Figures 1 and 4 . The first was probably the result of 


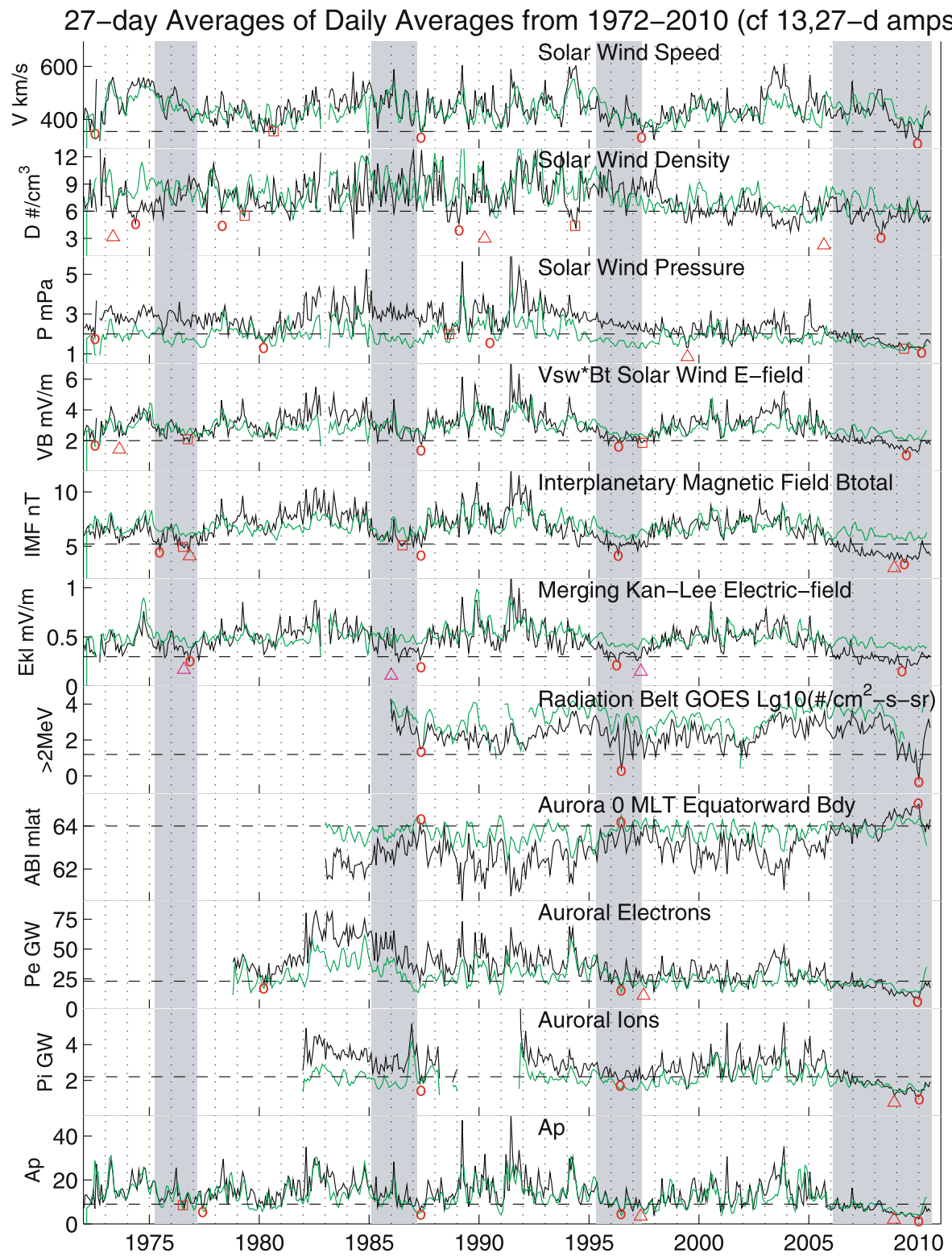

Figure 4 27-day (Bartels rotation) averages from 1972 to 2010 for various solar wind and geophysical parameters from Figure 1 in black and the 27-day averages of the sum of the 13.5- and 27-day amplitudes from Figures $2 \mathrm{~b}$ and $2 \mathrm{c}$ added to a baseline (day 48 in 2010) when the rotation amplitudes were minimal in dark green.

ICME activity and the second caused by HSS as suggested by the frequency of the solar wind structures plotted in Figure 1, although the location of the Earth at the highest heliolatitudes during equinox also contributed, as explained below. These $V_{\text {sw }}$ semiannual peaks are captured at lower amplitudes by the sum of the 13.5- and 27-day amplitudes. $D_{\text {sw }}$ also showed 


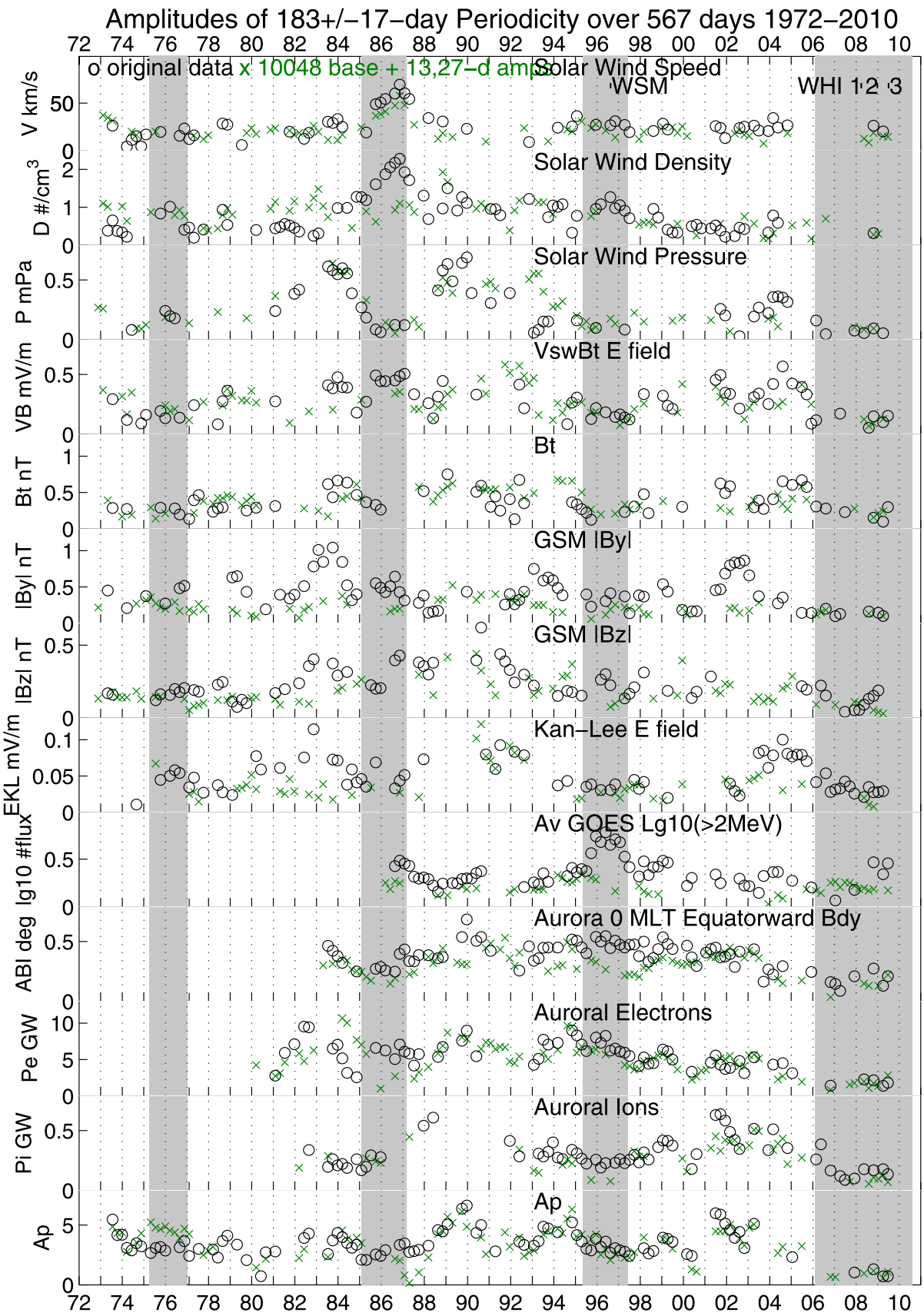

Figure 5 183-day semiannual amplitudes every 81 days in 569-day blocks for various solar wind and geophysical parameters using daily averages (black circles) and the sum of the 13.5- and 27-day amplitudes added to the baseline values from day 48 in 2010 (dark green $x$ 's), where both are plotted in Figure 4. Where the peaks are shorter than 166 days or longer than 200 days, the amplitudes are missing. 
large semiannual amplitudes at this time with solstice peaks, since $D_{\text {sw }}$ was anti-correlated with $V_{\text {sw }}$. However, the 13.5- and 27-day amplitudes did not capture the $D_{\text {sw }}$ semiannual amplitudes well because of significant amplitudes at higher frequencies in Figure 2a for $D_{\text {sw }}$. This solar minimum period was a time of very low tilt angle, and relatively flat HCS. The $\Phi$ (azimuth) angle in Figure 1 shows outward magnetic fields at the beginning of the year, and inward at the end of the year, where the highest velocities from coronal holes were encountered at the higher heliolatitudes in the axial equinoxes. The other relatively large semiannual amplitudes in 1986 in $\mathrm{VB}_{\mathrm{t}},\left|B_{y}\right|,\left|B_{z}\right|,>2 \mathrm{MeV}$ flux, $P_{\mathrm{e}}, \mathrm{ABI}$, and $A_{\mathrm{p}}$ usually showed lower semiannual amplitudes than the sum of the 13.5- and 27-day amplitudes. The semiannual amplitudes in 1996 are especially significant for $D_{\text {sw }}$ and $>2 \mathrm{MeV}$ electron number flux. The semiannual amplitudes in the present solar minimum are relatively small, especially in 2007.

Sometimes the peaks are at the solstices, which is true of $B_{\mathrm{t}}$ for Figure 4 with peaks in January 2004 and January 2005, and minor peaks in June 2004 and June 2005 leading to a strong semiannual variation in 2004-2005 in Figure 5. Luan et al. (2009) also studied the absence of the semiannual periodicity from 2002-2007 in $K_{\mathrm{p}}$ and in the electron hemispheric power $\left(\mathrm{HP}_{\mathrm{e}}\right)$ from the Global UltraViolet Imager (GUVI) onboard the NASA TIMED satellite. They found $K_{\mathrm{p}}$ and GUVI $\mathrm{HP}_{\mathrm{e}}$ to have peaks around the December solstice in 2003, 2004 and 2006, and in August in 2004 and 2005, which were associated with Kan-Lee merging electric field peaks in the same periods. The weak southward $B_{z}$ and $P_{\mathrm{sw}}$ in this period could also limit the regular modulation effects that contribute to peaks in the equinoxes.

However, most of the semiannual peaks in Figure 5 from the daily averages (black circles) are replicated at a lower level in the dark green $x$ 's, proving that most of the semiannual variations are due to variations in the 13.5- and 27-day amplitudes.

\section{Summary}

The above analysis indicates the following points, some of which are completely new, and others of which expand on previous findings:

i) HSS dominate the periodic structures in the descending and initial solar minimum phases, as shown by Emery et al. (2009), although the rotational periods are also present in the slow-speed wind. Solar rotation amplitudes minimized at the end of each solar minimum period and during the period of polarity change during solar maximum where $B_{\mathrm{t}}$ was also minimized. The rotational amplitudes were also high during SC 22 maximum, especially in 1991 when the active regions for CMEs were confined to a single solar longitude (Richardson, Mazur, and Mason, 1998), which imposed an additional 27-day periodicity to the transient activity.

ii) The shorter period 5-, 7-, 9- and 13.5-day amplitudes in most quantities were better correlated with each other than the 27-day amplitudes, and tended to maximize in the descending and early solar minimum phases with HSS. The strong 9-day periods in 2005 and 2008 and the 7-day periods in 2006 were also seen in $K_{\mathrm{p}}$, the neutral thermosphere density (Thayer et al., 2008; Lei et al., 2008a, 2008b, 2011), total electron content TEC (Lei et al., 2008c), neutral thermospheric polar winds (Lei et al., 2008c), and infrared $\mathrm{NO}$ and $\mathrm{CO}_{2}$ cooling (Mlynczak et al., 2008, 2010), and were absent or weak in the TIMED/SEE short wavelength radiation flux and $10.7 \mathrm{~cm}$ solar flux. 
iii) The ion auroral power input $P_{\mathrm{i}}$, although highly correlated with the electron auroral power input $P_{\mathrm{e}}$ and $A_{\mathrm{p}}$, showed different characteristics in its rotational amplitudes. The $P_{\mathrm{i}}$ amplitudes correlated best with the $A_{\mathrm{p}}$ amplitudes for the 5-, 7-, and 9-day periods, while the $P_{\mathrm{e}}$ amplitudes correlated best with the $A_{\mathrm{p}}$ amplitudes for the 13.5- and 27-day periods. Unlike most heliospheric and auroral parameters, the 13.5-day amplitudes of $P_{\mathrm{i}}$ were almost as poorly correlated with other parameters in Figure $3 \mathrm{~g}$ as the 27-day amplitudes. These results suggest that the processes governing ion precipitation into the atmosphere are significantly different than those for electrons. They both share pitch-angle scattering of diffuse precipitation, but they have different time constants. Protons also undergo charge exchange with ionospheric neutrals, and electrons experience monoenergetic and dispersive Alfvén wave acceleration mechanisms for discrete aurora. Our results also suggest that the auroral currents measured by the subauroral $A_{\mathrm{p}}$ and $K_{\mathrm{p}}$ indices are relatively sensitive to proton precipitation because the proton aurora is shifted $\sim 5^{\circ}$ equatorwards of the electron aurora in the dusk sector. The $A_{\mathrm{p}}$ currents appear to be sensitive to proton precipitation especially for 9-day and shorter periods, while the $A_{\mathrm{p}}$ currents are governed by electron precipitation for 13.5- and 27-day periodicities.

iv) The tilt in the HCS minimizes at the end of each solar minimum period. At these times, the solar magnetic field is dipolar and the current sheet is usually relatively flat, leading to strong semiannual variations in the azimuth of the magnetic field as the Earth goes below the current sheet in the early part of the year $\left(-7.2^{\circ}\right.$ on $\left.6 \mathrm{March}\right)$ and above the current sheet in the latter part of the year $\left(+7.2^{\circ}\right.$ on 6 September). These geometric enhancements can be greatly added to when HSS or ICME activity of various geoeffectiveness maximizes during equinoxes. Semiannual periodicities were strongest in the solar wind speed during the solar minimum period of 1986 . The solar wind parameters with semiannual periodicities during most years were Dsw and the absolute values of the IMF $\left|B_{y}\right|$ and $\left|B_{z}\right|$ components in GSM coordinates. The largest $D_{\mathrm{sw}}$ semiannual amplitudes were during solar minimum in 1986 and 1996, similar to $V_{\mathrm{sw}}$ amplitudes.

v) Geometrical effects enhance the IMF negative $B_{z}$ component in equinoxes and reduce it during the solstices through the Russell-McPherron effect (Russell and McPherron, 1973). The Kan-Lee merging electric field, which is sensitive to negative $B_{z}$ in GSM coordinates, had maximum semiannual amplitudes during the descending or maximum phases of the solar cycle usually where the Kan-Lee electric field was largest due to peaks in both $B_{t}$ and $V_{\mathrm{sw}}$. The semiannual periodicities were stronger in the Earth-based parameters like the radiation belt $>2 \mathrm{MeV}$ electrons, the auroral electron or ion powers, and $A_{\mathrm{p}}$ due to the Russell-McPherron effect as well as an additional reduction of the solar wind flow into the Earth's magnetosphere and polar-cap area during solstices (Cliver, Kamide, and Ling, 2000). These semiannual Earth-based amplitudes were primarily the result of enhanced equinoctial 27-day amplitudes.

vi) Most of the semiannual amplitudes and periods in the solar wind or Earth-based parameters were the result of equinoctial peaks in the amplitudes of solar rotation periods of 13.5- and 27-day.

In summary, the changing nature of the solar magnetic field and the path of the Earth through the associated solar wind structures had parallels and distinctions for the four solar cycles studied. These solar and interplanetary differences lead to profoundly different effects in the radiation belt, the Earth's aurora, and magnetic activity in the upper atmosphere.

Acknowledgements The original and the intercalibrated DMSP and NOAA satellite hemispheric power estimates were taken from the Coupling, Energetics, and Dynamics of Atmospheric Regions (CEDAR) 
Database, which is supported by the National Science Foundation (NSF). The Air Force Research Laboratory Auroral Boundary Index (ABI) was provided by the USAF Research Laboratory, Hanscom AFB, MA via the CEDAR Database. The hourly solar wind plasma and IMF data were taken from the OMNI-2 collection from the Space Physics Data Facility at the Goddard Space Flight Center managed by Dr. Natalia Papitashvili. B.A.E. is supported by the National Science Foundation at the High Altitude Observatory within the National Center for Atmospheric Research. I.G.R. is supported by the National Aeronautics and Space Administration (NASA) at the Goddard Space Flight Center.

\section{References}

Aellig, M.R., Lazarus, A.J., Steinberg, J.T.: 2001, The solar wind helium abundance: Variation with wind speed and the solar cycle. Geophys. Res. Lett. 28, 2767-2770.

Baker, D.N., McPherron, R.L., Cayton, T.E., Klebesadel, R.W.: 1990, Linear prediction filter analysis of relativistic electron properties at $6.6 R_{\mathrm{E}}$. J. Geophys. Res. 95(A9), $15133-15140$.

Chandra, S., Vats, H.O., Iyer, K.N.: 2009, Differential coronal rotation using radio images at 17 GHz. Mon. Not. Roy. Astron. Soc. 400, L34-L37.

Cliver, E.W., Kamide, Y., Ling, A.G.: 2000, Mountains versus valleys: Semiannual variation of geomagnetic activity. J. Geophys. Res. 105, 2413-2424.

Emery, B.A., Evans, D.S., Greer, M.S., Kadinsky-Cade, K., Holeman, E., Rich, F.J., Xu, W.: 2005, NOAA and DMSP intersatellite adjusted hemispheric power data sets, described at http://cedarweb.hao.ucar.edu/ wiki/index.php/Instruments:ehp in the Coupling, Energetics and Dynamics of Atmospheric Regions (CEDAR) Database at the National Center for Atmospheric Research (NCAR), Boulder, Colorado, USA (revised in 2007).

Emery, B.A., Evans, D.S., Greer, M.S., Holeman, E., Kadinsky-Cade, K., Rich, F.J., Xu, W.: 2006, The low energy auroral electron and ion hemispheric power after NOAA and DMSP intersatellite adjustments. NCAR Scientific and Technical Report, TN-470+STR (http://cedarweb.hao.ucar.edu/wiki/index.php/ Media:Str470.pdf in the CEDAR Database or available through the NCAR library at http://library.ucar. edu).

Emery, B.A., Coumans, V., Evans, D.S., Germany, G.A., Greer, M.S., Holeman, E., Kadinsky-Cade, K., Rich, F.J., Xu, W.: 2008, Seasonal, $K_{\mathrm{p}}$, solar wind, and solar flux variations in long-term single pass satellite estimates of electron and ion auroral hemispheric power. J. Geophys. Res. 113, A06311. doi:10.1029/ 2007JA012866.

Emery, B.A., Richardson, I.G., Evans, D.S., Rich, F.J.: 2009, Solar wind structure sources and periodicities of auroral electron power over three solar cycles. J. Atmos. Terr. Phys. 71. doi:10.1016/j.jastp.2008.08.005.

Fang, X., Liemohn, M.W., Kozyra, J.U., Evans, D.S., DeJong, A.D., Emery, B.A.: 2007a, Global 30-240 keV proton precipitation in the 17-18 April 2002 geomagnetic storms: 1. Patterns. J. Geophys. Res. 112, A05301. doi:10.1029/2006JA011867.

Fang, X., Ridley, A.J., Liemohn, M.W., Kozyra, J.U., Evans, D.S.: 2007b, Global 30-240 keV proton precipitation in the 17-18 April 2002 geomagnetic storms: 3. Impact on the ionosphere and thermosphere. J. Geophys. Res. 112, A07310. doi:10.1029/2006JA012144.

Fenimore, E.E., Asbridge, J.R., Bame, S.J., Feldman, W.C., Gosling, J.T.: 1978, The power spectrum of the solar wind speed for periods greater than 10 days. J. Geophys. Res. 83, $4353-4357$.

Fuller-Rowell, T.J., Evans, D.S.: 1987, Height-integrated Pedersen and Hall conductivity patterns inferred from the TIROS/NOAA satellite data. J. Geophys. Res. 92, 7606-7618.

Galand, M., Fuller-Rowell, T.J., Codrescu, M.V.: 2001, Response of the upper atmosphere to auroral protons. J. Geophys. Res. 106, $127-139$.

Gibson, S.E., Kozyra, J.U., de Toma, G., Emery, B.A., Onsager, T., Thompson, B.J.: 2009, If the Sun is so quiet, why is the Earth ringing? A comparison of two solar minimum intervals. J. Geophys. Res. 114(A9). doi:10.1029/2009JA014342.

Gosling, J.T., Asbridge, J.R., Bame, S.J., Feldman, W.C.: 1976, Solar wind speed variations: $1962-1974$. J. Geophys. Res. 81, $5061-5070$.

Gosling, J.T., Asbridge, J.R., Bame, S.J., Feldman, W.C.: 1977, Preferred solar wind emitting longitudes on the Sun. J. Geophys. Res. 82, 2371-2376.

Gussenhoven, M.S., Hardy, D.A., Burke, W.J.: 1981, DMSP/F2 electron observations of equatorward auroral boundaries and their relationship to magnetospheric electric fields. J. Geophys. Res. 86, $768-778$.

Gussenhoven, M.S., Hardy, D.A., Heinemann, N.: 1983, Systematics of the equatorward diffuse auroral boundary. J. Geophys. Res. 88, $5692-5708$.

Hardy, D.A., Gussenhoven, M.S., Brautigam, D.: 1989, A statistical model of auroral ion precipitation. J. Geophys. Res. 94, 370-392 1989. 
Hardy, D.A., Holeman, E.G., Burke, W.J., Gentile, L.C., Bounar, K.H.: 2008, Probability distributions of electron precipitation at high magnetic latitudes. J. Geophys. Res. 113, A06305. doi:10.1029/ 2007JA012746.

Howard, R.F.: 1996, Solar active regions as diagnostics of subsurface conditions. Annu. Rev. Astron. Astrophys. 34, 75-109.

Kan, J.R., Lee, L.C.: 1979, Energy coupling and the solar wind dynamo. Geophys. Res. Lett. $6,577$.

Knipp, D.J., Tobiska, W.K., Emery, B.A.: 2004, Solar direct and indirect thermospheric heating sources for solar cycles 21 - 23. Solar Phys. 224, $495-505$.

Lei, J., Thayer, J.P., Forbes, J.M., Sutton, E.K., Nerem, R.S.: 2008a, Rotating solar coronal holes and periodic modulation of the upper atmosphere. Geophys. Res. Lett. 35, L10109. doi:10.1029/2008GL033875.

Lei, J., Thayer, J.P., Forbes, J.M., Sutton, E.K., Nerem, R.S., Temmer, M., Veronig, A.M.: 2008b, Global thermospheric density variations caused by high-speed solar wind streams during the declining phase of solar cycle 23. J. Geophys. Res. 113, A11303. doi:10.1029/2008JA013433.

Lei, J., Thayer, J.P., Forbes, J.M., Wu, Q., She, C., Wan, W., Wang, W.: 2008c, Ionosphere response to solar wind high-speed streams. Geophys. Res. Lett. 35, L10105. doi:10.1029/2008GL035208.

Lei, J., Thayer, J.P., Wang, W., McPherron, R.L.: 2011, Impact of CIR storms on thermosphere density variability during the solar minimum of 2008. Solar Phys. 83. doi:10.1007/s11207-010-9563-y (this issue).

Lomb, N.R.: 1976, Least-squares frequency analysis of unequally spaced data. Astrophys. Space Sci. 39, $447-462$.

Luan, X., Wang, W., Burns, A.G., Solomon, S.C., Zhang, Y., Paxton, L.J.: 2009, Unusual declining phase of solar cycle 23: Weak semiannual variations of auroral hemispheric power and geomagnetic activity. Geophys. Res. Lett. 36, L22102. doi:10.1029/2009GL040825.

Maris, O., Maris, G.: 2005, Specific features of the high-speed plasma stream cycles. Adv. Space Res. 35, $2129-2140$.

Mende, S.B., Frey, H.U., Immel, T.J., Mitchell, D.G., C:son-Brandt, P., Gérard, J.-C.: 2002, Global comparison of magnetospheric ion fluxes and auroral precipitation during a substorm. Geophys. Res. Lett. 29(12), 1609. doi:10.1029/2001GL014143.

McComas, D.J., Ebert, R.W., Elliott, H.A., Goldstein, B.E., Gosling, J.T., Schwadron, N.A., Skoug, R.M.: 2008, Weaker solar wind from the polar coronal holes and the whole Sun. Geophys. Res. Lett. 35(18), L18103. doi:10.1029/2008GL034896.

McComas, D.J., Elliott, H.A., Schwadron, N.A., Gosling, J.T., Skoug, R.M., Goldstein, B.E.: 2003, The three-dimensional solar wind around solar maximum. Geophys. Res. Lett. 30, $1517-1520$.

Miyoshi, Y., Kataoka, R.: 2005, Ring current ions and radiation belt electrons during geomagnetic storms driven by coronal mass ejections and corotating interaction regions. Geophys. Res. Lett. 32, L21105. doi:10.1029/2005GL024590.

Mlynczak, M.G., Martin-Torres, F.J., Mertens, C.J., Marshall, B.T., Thompson, R.E., Kozyra, J.U., Remsberg, E.E., Gordley, L.L., Russell, J.M. III, Woods, T.: 2008, Solar-terrestrial coupling evidenced by periodic behavior in geomagnetic indexes and the infrared energy budget of the thermosphere. Geophys. Res. Lett. 35, L05808. doi:10.1029/2007GL032620.

Mlynczak, M.G., Hunt, L.A., Kozyra, J.U., Russell, J.M. III: 2010, Short-term periodic features observed in the infrared cooling of the thermosphere and in solar and geomagnetic indexes from 2002 to 2009. Proc. Roy. Soc. A 466, 3409 - 3419. doi:10.1098/rspa.2010.0077.

Mursula, K., Zieger, B.: 1996, The 13.5-day periodicity in the sun, solar wind, and geomagnetic activity: The last three solar cycles. J. Geophys. Res. 101, 27077-27090.

Newell, P.T., Sotirelis, T., Wing, S.: 2009, Diffuse, monoenergetic, and broadband aurora: The global precipitation budget. J. Geophys. Res. 114, A09207. doi:10.1029/2009JA014326.

Richardson, J.D., Kasper, J.C.: 2008, Solar cycle variations of solar wind dynamics and structures. J. Atmos. Terr. Phys. 70. doi:10.1016/j.jastp.2007.08.039.

Richardson, I.G., Cane, H.V., Cliver, E.W.: 2002, Sources of geomagnetic activity during nearly three solar cycles (1972-2000). J. Geophys. Res. 107(8). doi:10.1029/2001JA000504.

Richardson, I.G., Cliver, E.W., Cane, H.V.: 2000, Sources of geomagnetic activity over the solar cycle: Relative importance of coronal mass ejections, high-speed streams, and slow solar wind. J. Geophys. Res. 105, 18203 - 18213 .

Richardson, I.G., Mazur, J.E., Mason, G.M.: 1998, A comparison of recurrent energetic ion enhancements observed at Ulysses and at 1 AU by IMP 8 and SAMPEX: Ulysses launch until following the first north polar passage. J. Geophys. Res. 103, 2115.

Reiff, P.H., Luhmann, J.G.: 1986, Solar wind control of the polar-cap voltage. In: Kamide, Y., Slavin, J.A. (eds.) Proceedings of Solar Wind-Magnetosphere Coupling, Terra Scientific Publishing Company, Tokyo, $453-476$. 
Rostoker, G., Skone, S., Baker, D.N.: 1998, On the origin of relativistic electrons in the magnetosphere associated with some geomagnetic storms. Geophys. Res. Lett. 25, $3701-3704$.

Russell, C.T., McPherron, R.L.: 1973, Semiannual variation of geomagnetic activity. J. Geophys. Res. 78, 92-108.

Scargle, J.D.: 1982, Studies in astronomical time series analysis, II.Statistical aspects of spectral analysis of unevenly spaced data. Astrophys. J. 253, 835-853.

Sheeley, N.R. Jr., Harvey, J.W., Feldman, W.C.: 1976, Coronal holes, solar wind streams and recurrent geomagnetic disturbances - 1973-1976. Solar Phys. 49, 271.

Svalgaard, L., Wilcox, J.W.: 1975, Long term evolution of solar sector structure. Solar Phys. 41, 461 -475.

Thayer, J.P., Lei, J., Forbes, J.M., Sutton, E.K., Nerem, R.S.: 2008, Thermospheric density oscillations due to periodic solar wind high speed streams. J. Geophys. Res. 113, A06307. doi:10.1029/2008JA013190.

Turner, N.E., Cramer, W.D., Earles, S.K., Emery, B.A.: 2009, Geoefficiency and energy partitioning in CIRdriven and CME-driven storms. J. Atmos. Solar Ter. Phys. 71, $1023-1031$.

Vassiliadis, D., Klimas, A.J., Kanekal, S.G., Baker, D.N., Weigel, R.S.: 2002, Long-term-average, solar cycle, and seasonal response of magnetospheric energetic electrons to the solar wind speed. J. Geophys. Res. 107(A11), 1383. doi:10.1029/2001JA000506.

Wang, Y.-M., Sheeley, N.R. Jr., Rich, N.B.: 2000, Geophys. Res. Lett. 27, 149.

Wibberenz, G., Richardson, I.G., Cane, H.V.: 2002, A simple concept for modeling cosmic ray modulation in the inner heliosphere during solar cycles 20-23. J. Geophys. Res. 107(A11), 1343. doi:10.1029/ 2002JA009461.

Wolf, R.A.: 1995, Magnetospheric configuration. In: Kivelson, M.G., Russell, C.T. (eds.) Introduction to Space Physics, Cambridge University Press, Cambridge, 288-329.

Zhang, J., Richardson, I.G., Webb, D.F., Gopalswamy, N., Huttunen, E., Kasper, J.C., Nitta, N.V., Poomvises, W., Thompson, B.J., Wu, C.-C., Yashiro, S., Zhukov, A.N.: 2007, Solar and interplanetary sources of major geomagnetic storms (Dst $\leq-100 \mathrm{nT}$ ) during 1996-2005. J. Geophys. Res. 112, A10102. doi:10. 1029/2007JA012321. 